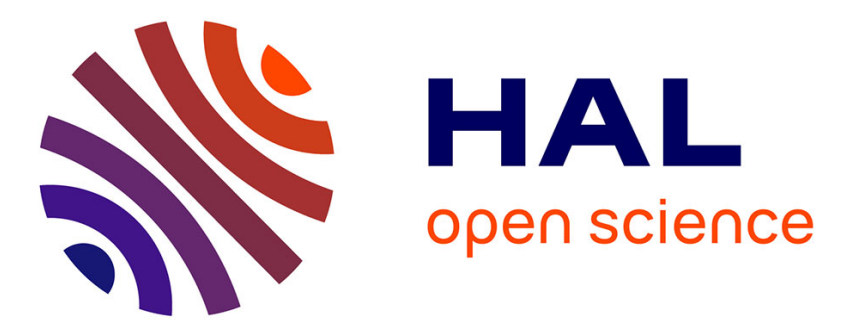

\title{
Exemples de croisements de deuxième espèce dans le diagramme d'énergie d'un atome habillé par des photons de radiofréquence
}

\author{
G. Grynberg, J. Dupont-Roc, S. Haroche, C. Cohen-Tannoudji
}

\section{- To cite this version:}

G. Grynberg, J. Dupont-Roc, S. Haroche, C. Cohen-Tannoudji. Exemples de croisements de deuxième espèce dans le diagramme d'énergie d'un atome habillé par des photons de radiofréquence. Journal de Physique, 1973, 34 (7), pp.537-558. 10.1051/jphys:01973003407053700 . jpa-00207415

\section{HAL Id: jpa-00207415 https://hal.science/jpa-00207415}

Submitted on 1 Jan 1973

HAL is a multi-disciplinary open access archive for the deposit and dissemination of scientific research documents, whether they are published or not. The documents may come from teaching and research institutions in France or abroad, or from public or private research centers.
L'archive ouverte pluridisciplinaire $\mathbf{H A L}$, est destinée au dépôt et à la diffusion de documents scientifiques de niveau recherche, publiés ou non, émanant des établissements d'enseignement et de recherche français ou étrangers, des laboratoires publics ou privés. 


\title{
EXEMPLES DE CROISEMENTS DE DEUXIÈME ESPẼCE DANS LE DIAGRAMME D'ÉNERGIE D'UN ATOME HABILLÉ PAR DES PHOTONS DE RADIOFRÉQUENCE
}

\author{
G. GRYNBERG, J. DUPONT-ROC, S. HAROCHE et C. COHEN-TANNOUDJI
}

Laboratoire de Spectroscopie Hertzienne de l'ENS, 24, rue Lhomond, 75005 Paris, France

(Reçu le 28 décembre 1972)

\begin{abstract}
Résumé. - Les résultats obtenus dans le précédent article, concernant l'existence de plusieurs types de croisements de niveaux, sont illustrés sur les diagrammes d'énergie d'atomes habillés par des photons de radiofréquence, de polarisations diverses.

Une étude détaillée des symétries du système global atome + radiofréquence permet de classer simplement tous les croisements de première espèce et certains croisements de deuxième espèce (liés à des symétries ponctuelles). A partir d'une formulation quantique du théorème de Majorana, on identifie les autres croisements comme étant des croisements de deuxième espèce de tous ordres (croisements de Majorana). On présente enfin une étude expérimentale des propriétés d'une résonance observable au voisinage d'un croisement de Majorana.

Abstract. - The results obtained in the preceding article concerning the existence of various types of level crossings are illustrated on the energy diagrams of atoms dressed by RF photons of various polarizations.

By studying the symmetries of the total system atom + RF field, one determines all the crossings of the first kind and some crossings of the second kind (related to point symmetries). Using a quantum formulation of Majorana's theorem, one identifies all the other crossings with crossings of the second kind of all orders (" Majorana's crossings »). Finally, an experimental study of the properties of a resonance observable near a Majorana's crossing is presented.
\end{abstract}

1. Introduction. - Nous avons, dans un précédent article [1], étudié et classé les différents types de croisements de niveaux susceptibles d'apparaître dans le diagramme d'énergie d'un système quantique lorsqu'on fait varier un paramètre $\omega_{0}$ dont dépend le hamiltonien He du système.

Nous avons vu que pour distinguer les différentes sortes de croisements se produisant entre deux niveaux $|\overline{\mathrm{a}}\rangle$ et $|\overline{\mathrm{b}}\rangle$, il est utile de considérer des expressions de la forme :

$$
\left\langle\overline{\mathrm{a}} \mid \frac{\mathrm{d} \overline{\mathrm{k}}_{1}}{\mathrm{~d} \omega_{0}}\right\rangle\left\langle\overline{\mathrm{k}}_{1} \mid \frac{\mathrm{d} \overline{\mathrm{k}}_{2}}{\mathrm{~d} \omega_{0}}\right\rangle \cdots\left\langle\overline{\mathrm{k}}_{n} \mid \frac{\mathrm{d} \overline{\mathrm{b}}}{\mathrm{d} \omega_{0}}\right\rangle .
$$

Chacune de ces expressions fait intervenir une chaîne d'états propres intermédiaires $\left|\overline{\mathrm{k}}_{1}\right\rangle,\left|\overline{\mathrm{k}}_{2}\right\rangle, \ldots$, $\mid \overline{\mathrm{k}}_{n}>$ de He et relie $\mid \overline{\mathrm{a}}>$ à $\mid \overline{\mathrm{b}}>$ par la succession des produits scalaires de chaque état avec la dérivée du suivant par rapport à $\omega_{0}$. Quand toutes les chaînes ainsi définies sont identiquement nulles, quelle que soit la suite d'états intermédiaires envisagée, les deux niveaux $|\bar{a}\rangle$ et $|\bar{b}\rangle$ varient, lorsqu'on balaie $\omega_{0}$, dans des sous-espaces orthogonaux. Ils sont alors de symétries différentes et le croisement de niveaux est dit de première espèce. Lorsque l'une des chaînes précédentes est non identiquement nulle, il n'est plus possible d'assigner une symétrie différente aux deux niveaux qui ne varient plus dans des sous-espaces stables orthogonaux. Le croisement est alors dit de deuxième espèce. Si toutes les chaînes faisant intervenir moins de $p$ états intermédiaires sont identiquement nulles et si une chaîne à $p$ états intermédiaires est effectivement différente de zéro, les deux niveaux $\mid \overline{\mathrm{a}}>$ et $\mid \overline{\mathrm{b}}>$ sont dits d'ordre relatif $p$ et le croisement de deuxième espèce qu'ils forment est d'ordre $p$. Nous avons enfin montré que certains croisements de deuxième espèce d'ordre zéro, tels que

$$
\left\langle\bar{a} \mid \frac{\mathrm{d} \bar{b}}{\mathrm{~d} \omega_{0}}\right\rangle \neq 0,
$$

peuvent être liés à une symétrie accidentelle du hamiltonien, existant au point de croisement $\omega_{0}=\omega_{0 \mathrm{c}}$ seulement et ne subsistant pas pour les autres valeurs du paramètre $\omega_{0}$.

La classification précédente est intéressante dans la mesure où, suivant la nature du croisement, les propriétés physiques du système au voisinage de $\omega_{0}=\omega_{0 \mathrm{c}}$ peuvent être très différentes. Nous avons montré en particulier, dans le cas d'un croisement de première 
espèce, que si le système se trouve initialement préparé dans l'état $|\overline{\mathbf{a}}\rangle$ pour une valeur $\omega_{0}<\omega_{0 \mathrm{c}}$ et si l'on balaie $\omega_{0}$ jusqu'au point de croisement et au-delà, le système ne peut, quelle que soit la vitesse du balayage, passer dans l'état $\mid \bar{b}>$; lorsqu'au contraire le croisement est de deuxième espèce, le passage à travers le point de croisement ne peut s'effectuer adiabatiquement (c'est-à-dire sans transition irréversible vers $|\bar{b}\rangle$ ) que si la variation du paramètre $\omega_{0}$ est suffisamment lente, la vitesse du balayage devant être d'autant plus faible que l'ordre du croisement de deuxième espèce est plus bas.

Une autre distinction intéressante entre croisements de première et de deuxième espèce apparaît lorsque le hamiltonien $\mathcal{H}$ du système peut se diviser naturellement en une partie non perturbée $\mathfrak{H}_{0}$, dont on connaît les états propres et les énergies, et une perturbation $V$, petite devant $\mathscr{H}_{0}$. Envisageons un croisement de première espèce de $\mathscr{H}_{0}$ entre deux niveaux $|\mathrm{a}\rangle$ et $|\mathrm{b}\rangle$ de symétries différentes, c'est-à-dire associés à des valeurs propres distinctes $l_{a}$ et $l_{b}$ d'un opérateur de symétrie $L$, indépendant de $\omega_{0}$ et commutant avec $\mathfrak{H}_{0}$. Si $V$ respecte la symétrie, c'est-à-dire si l'on a $[V, L]=0$, le couplage ne connecte à aucun ordre les niveaux $|\mathrm{a}\rangle$ et $|\mathrm{b}\rangle$, et les deux niveaux perturbés $|\overline{\mathrm{a}}\rangle$ et $\mid \bar{b}>$ qui se déduisent de $\mid \mathrm{a}>$ et $\mid \mathrm{b}>$ par branchement de la perturbation $V$ continuent à former un croisement de première espèce dans le diagramme d'énergie de $\mathcal{H}$. Pour exciter une cohérence entre ces niveaux et par voie de conséquence observer un signal de croisement de niveaux, nous avons vu qu'il est alors nécessaire d'exciter également une superposition linéaire des deux niveaux non perturbés $|\mathrm{a}\rangle$ et $|\mathrm{b}\rangle$, c'est-à-dire encore d'effectuer un pompage transversal dans la base des états propres non perturbés de $\mathfrak{H}_{0}$.

Supposons maintenant au contraire que $V$ ne respecte pas la symétrie $L([V, L] \neq 0)$. Les deux niveaux $|\mathrm{a}\rangle$ et $|\mathrm{b}\rangle$ peuvent alors être couplés par la perturbation, ce qui a en général pour effet de les repousser et de conduire à un anticroisement de niveaux de $H$. Nous avons vu cependant que, si le couplage entre $|\mathrm{a}\rangle$ et $|\mathrm{b}\rangle$ s'effectue indirectement par l'intermédiaire d'états $|\mathrm{i}\rangle,|\mathrm{j}\rangle$ différents de $|\mathrm{a}\rangle$ et $|\mathrm{b}\rangle$, il peut se trouver que le couplage s'annule juste au point de croisement sous l'effet d'une interférence destructive entre plusieurs processus. Les niveaux de $\mathcal{H}$, dont la symétrie par rapport à $L$ n'est plus définie, continuent alors à se croiser pour former un croisement de deuxième espèce. Pour exciter une cohérence entre les deux niveaux qui se croisent et détecter un signal au point de croisement, il n'est alors plus nécessaire d'effectuer un pompage cohérent dans la base des états non perturbés et une simple excitation des populations du système dans cette base est suffisante pour détecter le croisement de niveaux de Te. On observe alors un signal résonnant dont les propriétés sont, comme nous l'avons vu, intermédiaires entre celles des signaux de croisements de niveaux ordinaires (de première espèce) et des signaux d'anticroisements : comme pour un anticroisement, le signal correspond, lorsqu'on balaie $\omega_{0}$, à un transfert résonnant de populations entre des niveaux non perturbés de $H_{0}$; comme pour un signal de croisement, sa largeur tend vers zéro lorsque la largeur naturelle $\Gamma$ des niveaux d'énergie concernés s'annule (alors qu'il subsiste une largeur intrinsèque proportionnelle au couplage entre les niveaux qui s'anticroisent dans le cas d'une résonance d'anticroisement).

Le but du présent article est d'illustrer les considérations précédentes en étudiant les différents croisements de niveaux qui apparaissent dans le diagramme Zeeman d'un atome habillé [2] par des photons de radiofréquence. Rappelons que l'atome habillé est le système global constitué par un niveau atomique de spin $J$ en interaction avec un mode du champ électromagnétique quantifié. Le diagramme d'énergie d'un tel système présente un grand nombre d'anticroisements et de croisements de niveaux dont il est possible, comme nous allons le voir, de modifier à volonté le nombre et la position en variant la taille du spin $J$ du niveau atomique ou encore la polarisation de la radiofréquence. La grande richesse en croisements de niveaux de son diagramme d'énergie, ainsi que la possibilité de détecter ces croisements par des expériences simples de pompage optique, font ainsi de l'atome habillé un système quantique particulièrement bien indiqué pour la classification et l'étude des différents types de croisements de niveaux.

Le diagramme d'énergie de l'atome habillé se déduit de la connaissance de son hamiltonien $\mathscr{H}$ qui est constitué de trois termes.

- Un hamiltonien propre au système atomique placé dans un champ magnétique statique $H_{0}$, égal à $\omega_{0} J_{z}$ avec $\omega_{0}=-\gamma_{\alpha} H_{0}$ où $\gamma_{\alpha}$ est le rapport gyromagnétique du niveau $(\alpha J)$ considéré.

- Un hamiltonien propre au champ de radiofréquence $\omega a^{+} a$ où $\omega$ est la pulsation du champ de radiofréquence, $a$ et $a^{+}$étant respectivement les opérateurs d'annihilation et de création d'un photon dans le mode considéré.

- Un terme d'interaction entre l'atome et le champ égal à $\lambda \mathbf{J}\left(a \boldsymbol{\varepsilon}+a^{+} \boldsymbol{\varepsilon}^{*}\right)$ où $\boldsymbol{\varepsilon}$ est la polarisation du champ de radiofréquence et $\lambda$ un coefficient de couplage dont on peut montrer [2], [3] qu'il est égal à $\gamma_{\alpha} H_{1} / 2 \sqrt{\bar{n}}$ ( $H_{1}$ est l'amplitude du champ de radiofréquence classique et $\bar{n}$ le nombre moyen de photons dans le mode considéré). En tenant compte de ces trois termes, on obtient :

$$
\mathscr{H}=\omega_{0} J_{\mathbf{z}}+\omega a^{+} a+\lambda \mathbf{J}\left(a \varepsilon+a^{+} \varepsilon^{*}\right) .
$$

Les figures 1 et 2 donnent un aperçu du tracé des niveaux propres de $\mathcal{H}$ en fonction du champ magnétique $H_{0}$, pour diverses polarisations de la radiofréquence, et dans le cas respectivement d'un niveau atomique de $\operatorname{spin} J=\frac{1}{2}$ et $J=1$. Si l'on excepte deux 
cas de polarisation particulièrement simples (champs de radiofréquence tournant perpendiculaire à $H_{0}$, ou linéaire parallèle à $H_{0}$, figures $1 a, 2 a$ et $\left.1 b, 2 b\right)$, pour lesquels on peut diagonaliser rigoureusement $\mathfrak{H}$ [2], la détermination du diagramme d'énergie de l'atome habillé se fait par une méthode approchée. Si le champ de radiofréquence n'est pas trop intense, on a une allure assez bonne des niveaux d'énergie en considérant le terme $V$ d'interaction entre l'atome et le champ comme une perturbation vis-à-vis de :

$$
\mathscr{H}_{0}=\omega_{0} J_{\mathrm{z}}+\omega a^{+} a \text {. }
$$

Les états propres de $\mathcal{H}_{0}$, d'énergie $m \omega_{0}+n \omega$ sont simplement les états $|m, n\rangle$ représentant l'atome dans le sous-niveau Zeeman $m$ en présence de $n$ photons. Ces niveaux sont représentés par les lignes droites en pointillé sur les figures 1 et 2 . Ils constituent des diagrammes qui présentent une infinité de croisements de niveaux. Ces croisements apparaissent pour les

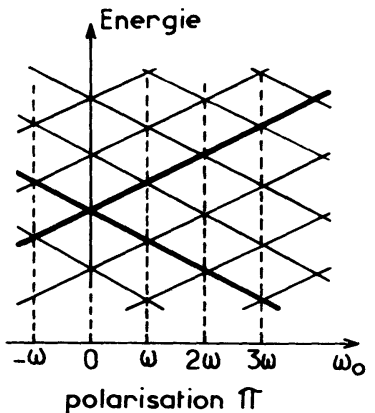

a)

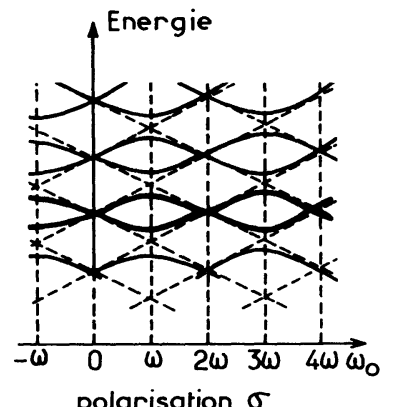

polarisation $\sigma$

c)

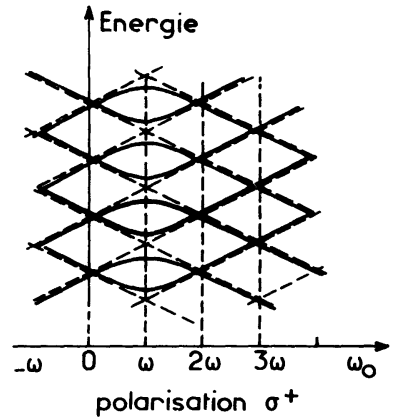

b)

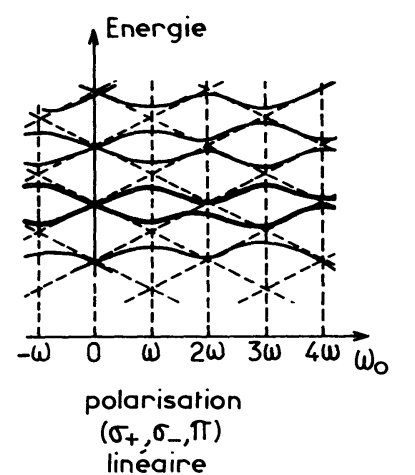

d)

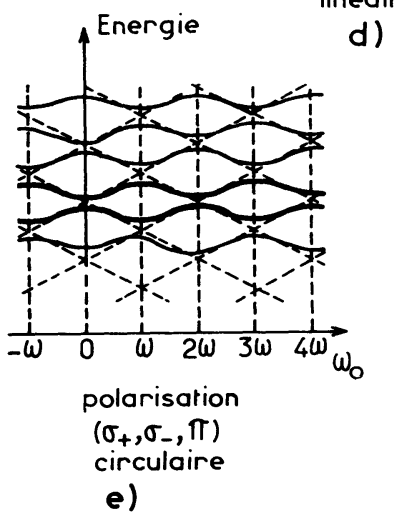

FIG. 1. - Variations en fonction du champ magnétique statique des niveaux d'énergie d'un spin $\frac{1}{2}$ habillé par des photons RF de diverses polarisations.

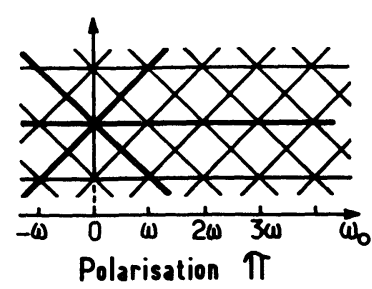

a)

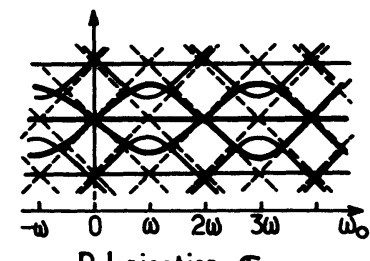

Polarisation $\sigma$

c) b)

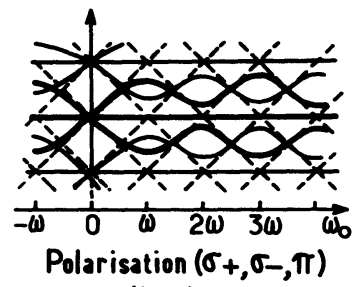

lineaire
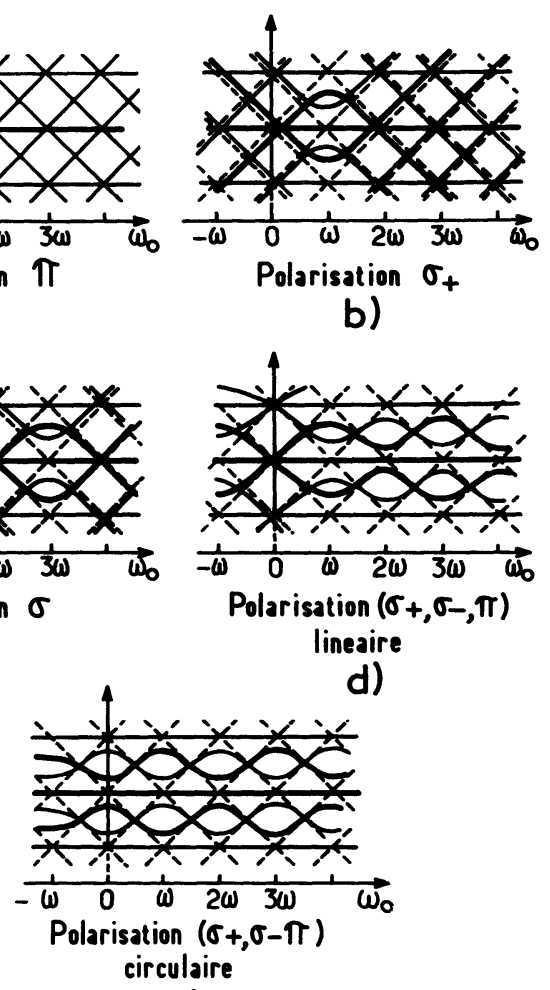

e)

Fig. 2. - Variations en fonction du champ magnétique statique des niveaux d'énergie d'un spin 1 habillé par des photons RF de même polarisation que dans la figure 1 .

valeurs du champ magnétique telles que $\omega_{0}=p \omega$ dans le cas d'un spin $\frac{1}{2}$ ( $p$ entier). Si le spin est supérieur à $\frac{1}{2}(J=1$ par exemple, voir Fig. 2), il existe, en plus des croisements précédents, des croisements dans des champs fractionnaires tels que $\omega_{0}=p \omega / q$ [croisements demi-entiers $\omega_{0}=(2 p+1) \omega / 2$ pour $\left.J=1\right]$. Quand on rajoute le terme de couplage $V$, la forme du diagramme d'énergie est modifiée : lorsque les niveaux qui se croisent se trouvent couplés entre eux directement ou indirectement par $V$, il apparaît un anticroisement de niveaux de $\mathcal{H}$. Les anticroisements ne peuvent provenir, comme nous le montrons plus loin, que des croisements entiers de $\mathcal{H}_{0}$, de la forme $\omega_{0}=p \omega$. Si les niveaux $|m, n\rangle$ et $\left|m^{\prime}, n^{\prime}\right\rangle$ de $\mathscr{H}_{0}$ qui se croisent ne sont au contraire pas couplés entre eux par la perturbation $V$, les niveaux de $\mathcal{H}$ continuent de se croiser, le point de croisement étant éventuellement déplacé par rapport à celui de $\mathscr{H}_{0}$. On obtient ainsi les configurations de niveaux des figures 1 et 2 qui ont été étudiés en détail dans des publications précédentes [2], [4].

Les différents croisements et anticroisements de niveaux apparaissant sur les diagrammes des figures 1 et 2 sont associés à divers types de résonance magnétique bien connus. Aux anticroisements $\omega_{0}=p \omega$ correspondent des résonances magnétiques à un ou plusieurs quanta [5]. Le système, préparé initialement dans l'état non perturbé $|m, n\rangle$, peut en effet, par suite du couplage résonnant entre les niveaux non 
perturbés, qui se traduit précisément par l'existence de l'anticroisement, se retrouver à un instant $t$ ultérieur dans un état $\left|m^{\prime}, n-p\right\rangle$. Ce processus correspond à une transition atomique $m \rightarrow m^{\prime}$ avec absorption de $p$ photons de radiofréquence. Les résonances correspondantes s'observent, comme toutes les résonances d'anticroisements, en pompage longitudinal dans la base non perturbée des états propres de $\mathscr{H}_{0}$ (on doit, par un procédé de préparation quelconque, peupler différemment les états atomiques $m$ et $m^{\prime}$ ce qui a pour effet de créer une différence de populations entre états $\mid m n>$ et $\left.\left|m^{\prime}, n-p\right\rangle\right)$. Toutes ces résonances d'anticroisements possèdent également la caractéristique importante d'avoir une largeur dépendant de l'intensité de la radiofréquence, qui ne s'annule pas lorsque la largeur naturelle $\Gamma$ des niveaux atomiques tend vers zéro.

Les croisements de niveaux de l'atome habillé sont associés quant à eux à des résonances magnétiques de cohérence d'un genre différent. Elles correspondent à la variation résonnante, lorsqu'on balaie $\omega_{0}$, d'une observable atomique possédant un élément de matrice non nul entre les états propres de l'atome habillé qui se croisent. Un certain nombre de ces croisements ont déjà fait l'objet d'études théoriques ou expérimentales au cours de ces dernières années. Il s'agit de divers croisements de niveaux observables sur un spin $\frac{1}{2}$ avec un champ de radiofréquence parallèle au champ statique (résonances paramétriques) [6] ou perpendiculaires au champ statique [7] (résonances $\left.\omega_{0}=2 p \omega\right)$ ou encore des croisements de niveaux observables sur un spin $\frac{1}{2}$ ou sur un spin 1 en présence d'un champ RF tournant perpendiculaire à $H_{0}$ [résonances $\omega_{0}=0$ et $\omega_{0}=2 \omega$ sur un spin $\frac{1}{2}$ [8] ; résonances $\omega_{0}=-\omega, \omega_{0}=0, \omega_{0}=\omega / 2, \omega_{0}=3 \omega / 2$, $\omega_{0}=2 \omega, \omega_{0}=3 \omega$ observables sur un spin 1 [2], $[9], \ldots$.$] ou encore des résonances de spectre demi-entier$ $\omega_{0}=(2 p+1) \omega / 2$ observables sur un spin $J>\frac{1}{2}$ avec un champ de radiofréquence en polarisation $\sigma[10]$. Toutes ces résonances ont pour caractéristique essentielle d'avoir une largeur intrinsèque proportionnelle à $\Gamma$, qui s'annule lorsque la durée de vie des niveaux devient infinie. Toutes ont pour leur observation nécessité l'utilisation d'un pompage atomique transversal (en excitant des cohérences atomiques entre états $\mid m>$ et $\mid m^{\prime}>$ différents, on prépare le système dans une superposition cohérente d'états propres non perturbés du hamiltonien $\mathfrak{J}_{0}$ ). Ce dernier point laisse supposer que les croisements de niveaux correspondants de l'atome habillé sont de première espèce.

La question qui se pose immédiatement est alors de savoir s'il en est de même de tous les croisements de niveaux observables sur les figures 1 et 2 . Nous allons montrer qu'il n'en est rien et qu'il existe en fait, suivant la taille du spin et la polarisation de la radiofréquence, des croisements de deuxième espèce de différents ordres. Alors que les croisements de première espèce sont constitués par des niveaux de symétries différentes qui ne peuvent se repousser, les niveaux qui constituent les croisements de deuxième espèce dans le diagramme de l'atome habillé n'ont pas de symétrie bien définie, et leur non-répulsion provient d'une interférence destructive au point de croisement entre plusieurs modes de couplage différents. Nous verrons qu'à ces croisements, qui sont en fait des anticroisements empêchés, sont associées des résonances magnétiques aux propriétés intermédiaires entre celles des transitions à plusieurs quanta et celles des résonances magnétiques de cohérence précédemment observées : d'une part, elles correspondent à un transfert résonnant de populations entre les niveaux atomiques sous l'effet du couplage avec la radiofréquence ; d'autre part, elles ne présentent par l'élargissement de radiofréquence caractéristique de transitions à plusieurs quanta.

Pour arriver à une classification simple des différents croisements de niveaux de l'atome habillé, il va nous falloir, pour commencer, faire une étude détaillée des symétries du système global atome + radiofréquence. Cette étude nous permettra de classer aisément les croisements observables sur un spin $\frac{1}{2}$ habillé (§2). Afin d'étendre la classification obtenue au cas d'un spin de taille quelconque, nous développerons ensuite une formulation quantique du théorème de Majorana $(\S 3)$. Nous préciserons alors les conditions d'observation des résonances de cohérence aux différents croisements de l'atome habillé, et nous décrirons quelques expériences nous ayant permis de vérifier les points prévus par la théorie $(\$ 4)$.

2. Etude des croisements de niveaux d'un spin $\frac{1}{2}$ habillé à partir des symétries du système global « atome + champ de radiofréquence $»$. - Pour diverses polarisations du champ de radiofréquence, le hamiltonien $\mathscr{H}=\mathscr{H}_{0}+V$ de l'atome habillé commute avec un certain opérateur indépendant du paramètre $\omega_{0}$; ceci traduit l'existence d'une symétrie du système atome + champ de radiofréquence indépendante du champ statique appliqué aux atomes. On peut alors associer un bon nombre quantique de symétrie à chacun des niveaux propres de $\mathcal{H}_{0}$ et de $\mathcal{H}$. Les différents croisements de niveaux de $\mathcal{H}_{0}$ correspondant à des nombres quantiques de symétrie différents restent des croisements de première espèce du hamiltonien complet He. S'il n'existe pas d'opérateur de symétrie commutant, quel que soit $\omega_{0}$, avec $\mathscr{H}$, mais si l'on peut trouver pour une valeur particulière $\omega_{0 c} \mathrm{du}$ paramètre $\omega_{0}$ un opérateur de symétrie entraînant une dégénérescence, les niveaux d'énergie de $\mathcal{H}$ se croisent nécessairement en $\omega_{0 c}$, formant un croisement de deuxième espèce dont il nous reste à préciser l'ordre. L'étude des diverses symétries de $\mathfrak{H}$, valables quel que soit le champ statique ou seulement pour certaines valeurs particulières de ce champ, se révèle donc utile pour répertorier l'ensemble des croisements de niveaux de première espèce et certains croisements de deuxième espèce dans les diagrammes d'énergie de l'atome habillé. Il se trouve d'ailleurs que l'on obtient ainsi l'ensemble des croisements apparaissant dans les 
diagrammes d'énergie d'un spin $\frac{1}{2}$ représentés sur la figure 1, et qui correspondent évidemment au cas le plus simple d'atome habillé que l'on puisse étudier.

2.1 LES SYMÉTRIES DU SYSTÈME GLOBAL SE CONSERVANT POUR TOUT $\omega_{0}$. - Pour certaines dispositions simples du champ statique $\mathbf{H}_{0}$ et du champ de radiofréquence $\mathbf{H}_{1}(t)$, le système global atome + radiofréquence présente une symétrie évidente. C'est le cas des polarisations $\pi, \sigma^{+}$et $\sigma$ de la radiofréquence :

2.1.1 Polarisation $\pi$ : invariance par rotation $d u$ système global autour de Oz. - Plaçons-nous tout d'abord dans le cas où l'on balaie le champ magnétique parallèlement au champ de radiofréquence (ces deux champs étant appliqués le long de $\mathrm{Oz}$; voir Fig. 3a). Dans ces conditions, toute rotation du système global autour de $\mathrm{Oz}$ est manifestement une opération de symétrie. Ce résultat évident peut se vérifier en remarquant que le champ de radiofréquence, dont le moment cinétique le long de $\mathrm{Oz}$ est nul, reste invariant dans une telle symétrie et que le hamiltonien $\mathcal{H}_{\pi}=\omega a^{+} a+\omega_{0} J_{\mathrm{z}}+\lambda J_{\mathrm{z}}\left(a+a^{+}\right)$commute avec la composante du moment cinétique atomique $J_{z}$, quel que soit $\omega_{0}$ :

$$
\left[\mathcal{H}_{\pi}, J_{\mathrm{z}}\right]=0 \text {. }
$$

Comme conséquence de cette symétrie, $m$ est un bon nombre quantique. Ainsi, dans le cas d'une polarisation $\pi$ de la radiofréquence, les croisements entre niveaux de $\mathcal{H}_{0}$ de $m$ différents restent tous des croisements de première espèce de $\mathcal{H}$. Il s'agit en fait de tous les croisements de niveaux $\omega_{0}=p \omega$ de la figure $1 a$. L'interprétation physique de ce résultat est très claire et tient à la conservation du moment

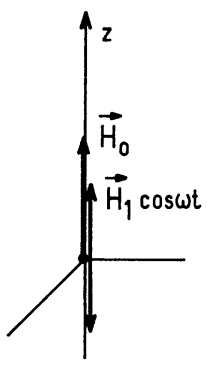

a)

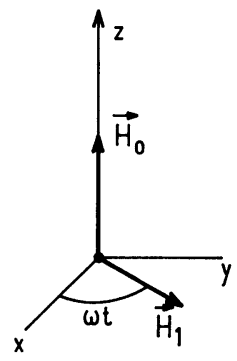

b)

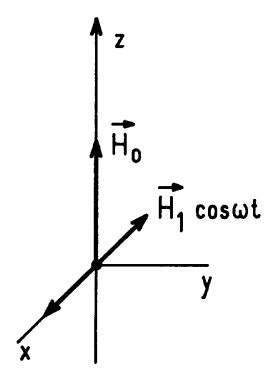

c)
Fig. 3. - Disposition relative du champ magnétique statique $\mathbf{H}_{0}$ et du champ RF $\mathbf{H}_{1}$, a) Polarisation $\pi$. b) Polarisation $\sigma^{+}$. c) Polarisation $\sigma$.

cinétique du système global. Les deux états propres $\mid+\frac{1}{2}, n>$ et $\mid-\frac{1}{2}, n+p>$ de $\mathscr{H}_{0}$ qui se croisent en $\omega_{0}=p \omega$ ne peuvent être couplés par aucun processus à plusieurs quanta, puisque les $p$ photons qui seraient absorbés ou émis lors d'un tel processus, et qui transportent un moment cinétique nul, ne pourraient fournir l'unité de moment angulaire nécessaire à la transition atomique $\Delta m=1$.
Remarque. - Le fait que tous les croisements de niveaux de l'atome habillé sont, pour cette polarisation de la radiofréquence, de première espèce apparaît clairement si on considère l'ordre relatif des niveaux : les états propres du système global ne dépendent pas en effet, dans ce cas, de l'amplitude du champ statique [2]. Il en résulte que toutes les quantités de la forme $\left\langle\bar{a} \mid \frac{d \bar{b}}{d \omega_{0}}\right\rangle$ sont identiquement nulles, et que deux niveaux quelconques de l'atome habillé (en particulier ceux qui se croisent) sont d'ordre relatif infini.

2.1.2 Polarisation $\sigma_{+}$: invariance par rotation $d u$ système global autour de $\mathrm{Oz}$. - Il existe un autre cas d'invariance de l'atome habillé par rotation autour de l'axe $\mathrm{Oz}$ : c'est celui où le champ de radiofréquence tourne à la vitesse angulaire $\omega$ dans un plan perpendiculaire à $\mathbf{H}_{0}$, aligné lui-même le long de $\mathrm{Oz}$ (voir Fig. $3 b$ ). Ce résultat physiquement évident se vérifie en constatant que le hamiltonien

$$
\mathcal{H}_{\sigma_{+}}=\omega_{0} J_{z}+\omega a^{+} a+\lambda\left(a J_{+}+a^{+} J_{-}\right)
$$

commute avec le moment cinétique $J_{\mathrm{z}}+a^{+} a$ (en effet $a^{+} a$, c'est-à-dire le nombre de photons de radiofréquence, n'est autre que le moment cinétique du champ habillant, puisque chaque photon $\sigma_{+}$a un moment cinétique +1 le long de $\mathrm{Oz}$ ) :

$$
\left[\mathcal{H}_{\sigma_{+}}, J_{z}+a^{+} a\right]=0
$$

comme conséquence de cette symétrie, $m+n$ est un bon nombre quantique dans le cas d'une polarisation $\sigma_{+}$de la radiofréquence. Il en résulte que les croisements de niveaux de $\mathscr{H}_{0}$ de $(m+n)$ différents restent des croisements de première espèce de l'atome habillé. Les croisements s'effectuant tous sur un spin $\frac{1}{2}$ entre niveaux tels que $\Delta m=+1$, tous les croisements de $\mathscr{H}_{0}$ tels que $\Delta n \neq 1$, c'est-à-dire tels que $\omega_{0} \neq \omega$, sont donc des croisements de première espèce dans le diagramme d'énergie de la figure $1 b$. Il s'agit en fait de tous les croisements de niveaux du diagramme. L'existence de ces croisements de niveaux de première espèce résulte ici encore de la nécessité de conserver le moment cinétique du système global au cours des transitions de radiofréquence: chaque photon $\sigma_{+}$ transportant une unité +1 de moment angulaire, le seul processus de couplage possible correspond à l'absorption d'un photon avec transition de l'atome de l'état $-\frac{1}{2}$ à $+\frac{1}{2}$. Ainsi, seuls les niveaux $\mid-\frac{1}{2}, n>$ et $\left|+\frac{1}{2}, n-1\right\rangle$ peuvent être couplés entre eux (résonance magnétique ordinaire), et tous les croisements entre niveaux de $\mathscr{H}_{0} \mid-\frac{1}{2}, n>$ et $\mid+\frac{1}{2}, n-p>$ avec $p \neq 1$ doivent rester de première espèce en présence du couplage $V$.

REMARQUE. - On peut traduire les considérations de symétrie précédentes en termes d'ordre relatif des niveaux : seuls les états de même valeur de $(m+n)$ étant couplés par $V, m+n$ reste un bon nombre quantique pour les états propres de $H$. Les états propres associés à des valeurs de $(m+n)$ différents 
sont d'ordre relatif infini. Les deux états associés à la même valeur de $(m+n)\left(m= \pm \frac{1}{2}\right)$ forment un anticroisement en $\omega_{0}=\omega$, et sont donc d'ordre relatif zéro.

2.1.3 Polarisation $\sigma$ : invariance par réflexion $d u$ système global par rapport au plan $\mathrm{xOz}$. - Etudions maintenant le cas d'un champ de radiofréquence linéaire $\mathbf{H}_{1}$ parallèle à $\mathrm{Ox}$, et donc perpendiculaire au champ statique $\mathbf{H}_{0}$ dirigé le long de $\mathrm{Oz}$ (Fig. $3 c$ ). S'il l'on fait une symétrie par rapport au plan xOy perpendiculaire à $\mathbf{H}_{0}$ (et qui contient donc $\mathbf{H}_{1}$ ) $J_{z}$, vecteur axial, reste invariant et en conséquence le hamiltonien non perturbé $\mathcal{H}_{0}=\omega_{0} J_{\mathrm{z}}+\omega a^{+} a$ ne change pas non plus, puisque le nombre de photons ne varie pas dans cette opération. Par contre, $J_{x}$ se change en $-J_{x}$; mais comme $H_{1}$ se transforme manifestement en $-\mathbf{H}_{1}$, le terme de couplage $V=-\gamma_{\alpha} H_{1 \mathrm{x}} J_{\mathrm{x}}=\lambda J_{\mathrm{x}}\left(a+a^{+}\right)$est invariant lui aussi dans cette réflexion. Nous montrons en fait dans l'Appendice I que l'opérateur de l'espace des états associé à la réflexion que nous venons de décrire, et qui laisse invariant le hamiltonien $\mathscr{H}$, est l'opérateur

$$
S=\mathrm{i}^{2 J} \mathrm{e}^{-\mathrm{i} \pi\left(J_{\mathrm{z}}-a^{+} a\right)} .
$$

On vérifie facilement que l'état non perturbé $\mid J, m, n>$ est état propre de $S$ associé à la valeur propre $(-1)^{J-m+n}$. On en déduit que, dans le cas d'une polarisation $\sigma$ de la radiofréquence, les croisements entre des niveaux de $\mathscr{H}_{0}$ correspondant à des parités différentes de $J-m+n$ sont associés à des croisements de niveaux de première espèce de $\mathcal{H}$. Comme les niveaux de $\mathcal{H}_{0}$ qui se croisent pour un spin $\frac{1}{2}$ correspondent à un $\Delta m= \pm 1$, on en conclut que tous les croisements tels que $\Delta n$ est pair, c'est-à-dire tels que l'on ait $\omega_{0}=2 p \omega$, sont des croisements de première espèce dans le diagramme d'énergie de la figure $1 c$. Il s'agit en fait de tous les croisements de niveaux de ce diagramme. L'existence de ces croisements de première espèce peut encore se déduire de la règle de conservation du moment cinétique du système global : chaque photon de polarisation $\sigma$ (qui peut s'analyser en deux composantes $\sigma_{+}$et $\sigma_{-}$tournant en sens inverse) transporte en effet une unité \pm 1 de moment angulaire le long de Oz. Seul un nombre impair de processus d'absorption ou d'émission de photons permet donc de réaliser une transition atomique $\Delta m= \pm 1$ entre les états $+\frac{1}{2}$ et $-\frac{1}{2}$. On comprend ainsi que les états $\mid-\frac{1}{2}, n>$ et $\mid+\frac{1}{2}, n-2 p>$ ne puissent être couplés à aucun ordre.

REMARQUE. - Ici encore, les considérations de symétrie peuvent se traduire en termes d'ordre relatif des niveaux ; $(-1)^{J-m+n}$ est en effet un bon nombre quantique non seulement pour les états non perturbés, mais aussi pour les niveaux propres de He Ce nombre quantique à associer à un niveau donné s'obtient aisément en déterminant celui du niveau non perturbé correspondant en champ nul, lorsqu'on débranche le couplage entre l'atome et le champ (voir Fig. 1c).
Si le niveau en question tend vers l'état $\mid+\frac{1}{2}, n>$, sa symétrie est alors $(-1)^{n}$; s'il tend vers l'état $\mid-\frac{1}{2}, n>$, sa symétrie est égale à $(-1)^{n+1}$. On appellera respectivement $\mid \overline{+\frac{1}{2}, n}>$ et $\mid \overline{-\frac{1}{2}, n}>$ ces niveaux (voir $\S$ suivant). Il est clair alors que deux niveaux $\left|\overline{+\frac{1}{2}, n}\right\rangle$ et $\left|\overline{-\frac{1}{2}, n^{\prime}}\right\rangle$ sont de symétries différentes si $n-n^{\prime}$ est pair alors que $\mid \overline{+\frac{1}{2}, n}>$ et $\overline{1+\frac{1}{2}, n^{\prime}}>$ sont de symétries différentes si $n-n^{\prime}$ est impair. Ces couples de niveaux sont d'ordre relatif infini. Au contraire, $\mid \overline{+\frac{1}{2}, n}>$ et $\mid \overline{-\frac{1}{2}, n^{\prime}}>\sin -n^{\prime}$ est impair, $\mid \overline{+\frac{1}{2}, n}>$ et $\mid \overline{+\frac{1}{2}, n^{\prime}}>$ si $n-n^{\prime}$ est pair, sont de même symétrie. On montre en appendice II qu'ils sont alors, quel que soit $n-n^{\prime}$, d'ordre relatif zéro.

Une étude détaillée des autres polarisations possibles de la radiofréquence (superposition cohérente de photons $\sigma$ et $\pi$ ) montre que l'on ne peut pas trouver d'autres observables $L$ commutant pour toute valeur du champ avec le hamiltonien $\mathcal{H}$. L'ordre relatif de deux niveaux du spin $\frac{1}{2}$ habillé est en effet pour une telle polarisation égal à zéro, quel que soit le couple de niveaux envisagé (voir démonstration en Appendice II). Il en résulte que nous venons de passer en revue tous les types possibles de croisements de première espèce dans le diagramme d'énergie d'un spin $\frac{1}{2}$. Dans le cas d'un champ de radiofréquence polarisé dans une direction faisant un angle quelconque avec $\mathbf{H}_{0}$ (Fig. 1d) ou encore d'un champ tournant dans un plan quelconque non perpendiculaire à $\mathbf{H}_{0}$ (Fig. 1e) aucune règle de symétrie n'empêche les niveaux qui se croisent dans le diagramme d'énergie de $\mathscr{H}_{0}$ d'être couplés par $V$ : en fait, tous les croisements de ces diagrammes, deviennent des anticroisements associés à des processus de transition à plusieurs quanta, sauf le croisement de niveaux en champ nul $\left(\omega_{0}=0\right)$ dans le cas d'un champ de radiofréquence polarisé linéairement (Fig. 1d). Nous montrons à présent qu'il s'agit là d'un croisement de niveaux de deuxième espèce d'ordre zéro, lié à l'existence d'une symétrie accidentelle par renversement du temps du hamiltonien $\mathcal{H}$ en champ nul.

2.2 UNE SYMÉTRIE « ACCIDENTELLE 》 DU SYSTÈME GLOBAL : RENVERSEMENT DU TEMPS EN CHAMP STATIQUE NUL POUR UN CHAMP DE RADIOFRÉQUENCE POLARISÉ LINÉAIREMENT. - Il est clair que lorsque le champ de radiofréquence est linéaire, dans une direction faisant un angle différent de 0 et $\pi / 2$ avec $\mathbf{H}_{0}$, aucune règle de sélection sur le moment cinétique n'interdit aux deux niveaux $\mid+\frac{1}{2}, n>$ et $\mid-\frac{1}{2}, n>$ qui se croisent en champ nul d'être couplés par $V$. La polarisation de chaque photon peut en effet être analysée comme une superposition cohérente des trois types de polarisation $\sigma_{+}, \sigma_{-}$et $\pi$, et l'on peut en fait, grâce à l'absorption ou l'émission stimulée de l'une ou l'autre de ces composantes, envisager quatre voies de diffusion du second ordre permettant de coupler l'état $\mid+\frac{1}{2}, n>$ à l'état $\mid-\frac{1}{2}, n>$. Ces quatre voies sont schématisées sur la figure 4 et correspondent à des processus de 


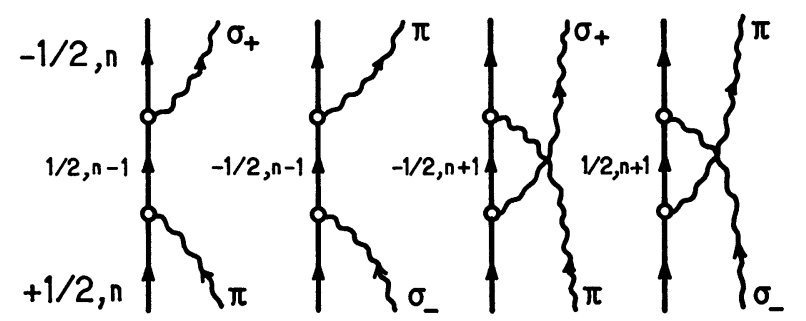

Fig. 4. - Schématisation des 4 amplitudes de diffusion permettant de relier l'état $\left|+\frac{1}{2}, n\right\rangle$ à l'état $\left|-\frac{1}{2}, n\right\rangle$. Pour $\omega_{0}=0$, ces 4 amplitudes interfèrent destructivement.

types Raman au cours desquels un photon de radiofréquence se trouve être absorbé puis réémis (ou inversement). Cependant on constate par le calcul [2] que les amplitudes de probabilité associées à ces quatre processus interfèrent de façon destructive, ce qui empêche les niveaux $\left|-\frac{1}{2}, n\right\rangle$ et $\left|+\frac{1}{2}, n\right\rangle$ de former un anticroisement. Il se trouve en fait, comme nous allons le voir, que la non-répulsion des niveaux est liée dans ce cas à une symétrie par renversement du temps du système en champ statique nul. Les niveaux qui continuent à se croiser ne peuvent alors constituer qu'un croisement de niveaux de deuxième espèce qui ne peut être que d'ordre zéro.

L'opérateur antiunitaire $T$ de renversement du temps agissant sur le système global atome + radiofréquence est décrit en Appendice I. On montre que, dans le cas d'une polarisation linéaire de la radiofréquence, il anticommute avec les opérateurs $a$ et $a^{+}$

$$
\mathrm{TaT}^{+}=-a ; \quad \mathrm{Ta}^{+} T^{+}=-a^{+} .
$$

Il est clair d'autre part que $T$ et $\mathcal{H}$ ne peuvent commuter en champ statique non nul puisque le terme Zeeman atomique $\omega_{0} J_{z}$ qui apparaît dès que $\mathbf{H}_{0} \neq 0$ anticommute en fait avec $T$ d'après la relation générale satisfaite par le moment cinétique :

$$
T \mathbf{J} T^{+}=-\mathbf{J} .
$$

En champ $H_{0}$ nul, par contre, le hamiltonien de l'atome habillé s'écrit :

$$
\mathscr{H}\left(\omega_{0}=0\right)=\omega a^{+} a+\lambda \mathbf{J} \boldsymbol{\varepsilon}\left(a+a^{+}\right)
$$

où $\varepsilon$ est le vecteur polarisation réel de la radiofréquence.

Compte tenu des relations (2.3) et (2.4), on déduit aisément que l'on a

$$
\left[T, \mathscr{H}\left(\omega_{0}=0\right)\right]=0
$$

ce qui établit la symétrie du hamiltonien par renversement du temps en champ nul. Cette symétrie entraîne une dégénérescence des niveaux de l'atome habillé en champ nul. Le système global étudié est en effet constitué d'un fermion (spin $\frac{1}{2}$ ), en présence d'un certain nombre de bosons (photons). C'est donc un système à nombre impair de fermions auquel on peut en champ $H_{0}$ nul, appliquer le théorème de Kramers qui stipule que tous les niveaux doivent être au moins doublement dégénérés.
Dès que $\mathbf{H}_{0} \neq 0$, la dégénérescence peut être levée ce qui explique que les niveaux de l'atome habillé se croisent en fait en champ nul. L'ordre de ce croisement est naturellement zéro, puisque tous les niveaux de l'atome habillé sont, dans ce cas de polarisation cohérente, d'ordre relatif zéro. Il est d'ailleurs facile de vérifier que les deux niveaux qui se croisent en champ nul sont bien d'ordre zéro puisque ces deux niveaux forment un anticroisement au voisinage du champ $\omega_{0}=2 \omega$ (voir Fig. $1 d$ ).

Ainsi se trouvent expliqués tous les croisements de niveaux apparaissant dans le diagramme d'énergie d'un spin $\frac{1}{2}$.

REMARQUE. - Si la radiofréquence a une polarisation circulaire (ou elliptique), l'atome habillé ne possède plus la symétrie par renversement du temps en champ nul. En effet, le sens de rotation de la radiofréquence est inversé par renversement du temps, ce qui revient à changer sa polarisation et, par voie de conséquence, le hamiltonien $\mathcal{H}$. La dégénérescence des niveaux en champ nul n'est alors plus imposée. En particulier lorsque la radiofréquence correspond à une polarisation cohérente, superposition de photons $\sigma_{+}, \sigma_{-}, \pi$, les quatre voies de diffusion symbolisées sur la figure 4 existent comme pour un champ polarisé linéairement, et aucun argument de symétrie ne conduit plus les amplitudes de transition correspondantes à s'annuler : on observe alors un anticroisement des niveaux en champ nul dont l'existence se révèle par l'apparition d'une résonance magnétique de type Raman en champ nul [11].

3. Etude des croisements de niveaux d'un spin $\mathbf{J}>1 / 2$ habillé, à partir d'une formulation quantique du théorème de Majorana. - 3.1 LE PROBLÈME DES CROISEMENTS DE NIVEAUX DE DEUXIÈME ESPÈCE POUR UN SPIN $J>\frac{1}{2}$ HABILLÉ PAR UN CHAMP DE RADIOFRÉQUENCE. - Les symétries du système global atome + radiofréquence que nous avons étudiées au paragraphe précédent sont valables quelle que soit la taille du spin $J$ du niveau car elles ne dépendent que de la forme du hamiltonien $\mathscr{H}$ naturellement indépendante de la grandeur de $J$. Cependant, dès que $J>\frac{1}{2}$, il est impossible de conclure au croisement ou au noncroisement de deux niveaux d'énergie de $\mathcal{H}_{0}$ à partir de l'examen des seules symétries de $\mathcal{H}$. Ce résultat apparaît clairement si l'on considère les diagrammes d'énergie d'un spin $J=1$ reproduits sur la figure 2 :

3.1.1 Lorsque le champ de radiofréquence a une polarisation cohérente, superposition de photons $\sigma_{+}, \sigma_{-}$et $\pi$, il n'existe aucune opération laissant invariante le hamiltonien du système. En champ statique non nul, aucune règle de sélection liée à une symétrie permanente (valable quel que soit $\omega_{0}$ ), ni aucune dégénérescence accidentelle liée à une symétrie ponctuelle (valable pour une valeur donnée de $\omega_{0}$ ) n'empêche donc deux niveaux de $\mathscr{H}_{0}$ qui se croisent de se repousser sous l'effet du couplage $V$. Il se trouve 
cependant que certains niveaux continuent à se croiser lorsqu'on applique ce terme de couplage. C'est le cas par exemple de tous les niveaux qui se coupent aux croisements non entiers de la forme $\omega_{0}=(2 p+1) \omega / 2$. La non-répulsion des niveaux en ces points peut être constatée en remarquant que le couplage éventuel entre les niveaux résulterait d'une interférence entre plusieurs chemins qui se révèle être complètement destructive. Ainsi les niveaux $|+1, n\rangle$ et $1-1, n+3\rangle$ qui se croisent dans le champ $\omega_{0}=3 \omega / 2$ peuvent être couplés via les deux chemins symbolisés sur la figure 5 ; on peut montrer [2] que les amplitudes de probabilités associées sont opposées :
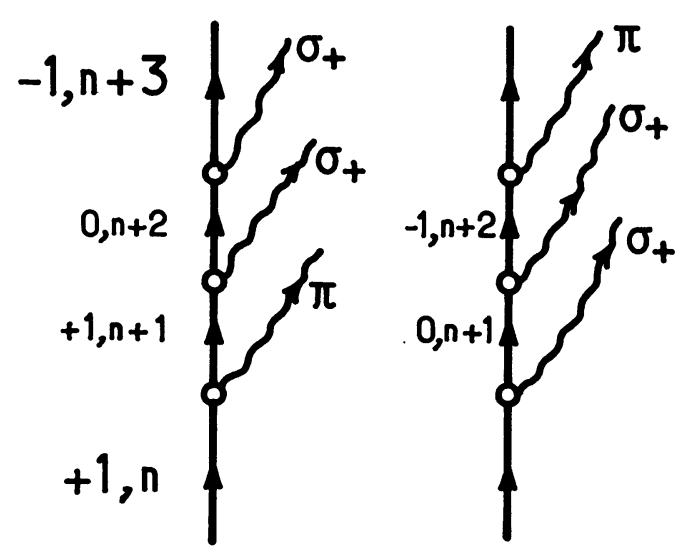

Fig. 5. - Schématisation des deux amplitudes de diffusion permettant de relier l'état $|+1, n\rangle$ à l'état $|-1, n+3\rangle$. Pour $\omega_{0} \simeq 3 \omega / 2$, ces deux amplitudes interfèrent destructivement.

3.1.2 Qui plus est, même s'il existe une symétrie du hamiltonien $\mathcal{H}$, deux niveaux de $\mathcal{H}_{0}$ de même symétrie peuvent continuer à se croiser lorsqu'on applique la perturbation $V$. Dans le cas d'un champ de radiofréquence en polarisation $\sigma$, nous avons vu qu'à chaque niveau de $\mathfrak{H}_{0}$ peut être associé un bon nombre quantique égal à $(-1)^{J-m+n}$. Les deux niveaux de $\mathfrak{H}_{0}, \mid+1, n>$ et $\mid-1, n+4>$, qui se croisent dans le champ $\omega_{0}=2 \omega$ (Fig. $2 c$ ), sont manifestement de même symétrie puisque $m$ et $n$ sont changés respectivement de deux et quatre unités lorsqu'on passe d'un niveau à l'autre. Pourtant ces deux niveaux ne se repoussent pas et continuent à se croiser dans le diagramme d'énergie de $\mathscr{H}$ (avec également le niveau $|0, n\rangle$; voir Fig. $2 c$ ). Le croisement est lié ici encore à une interférence destructive [4] entre les deux modes de couplage représentés par les diagrammes de la figure 6.

Il est donc clair que les considérations de symétrie, qui suffisent à justifier l'existence des différents types de croisement pour un spin $J=\frac{1}{2}$ habillé, ne permettent pas à elles seules de conclure à l'existence de ces croisements dès que $J>\frac{1}{2}$. Il apparaît en fait dans les diagrammes d'énergie une infinité de croisements nouveaux de deuxième espèce, qui résultent d'une interférence destructive entre plusieurs modes de
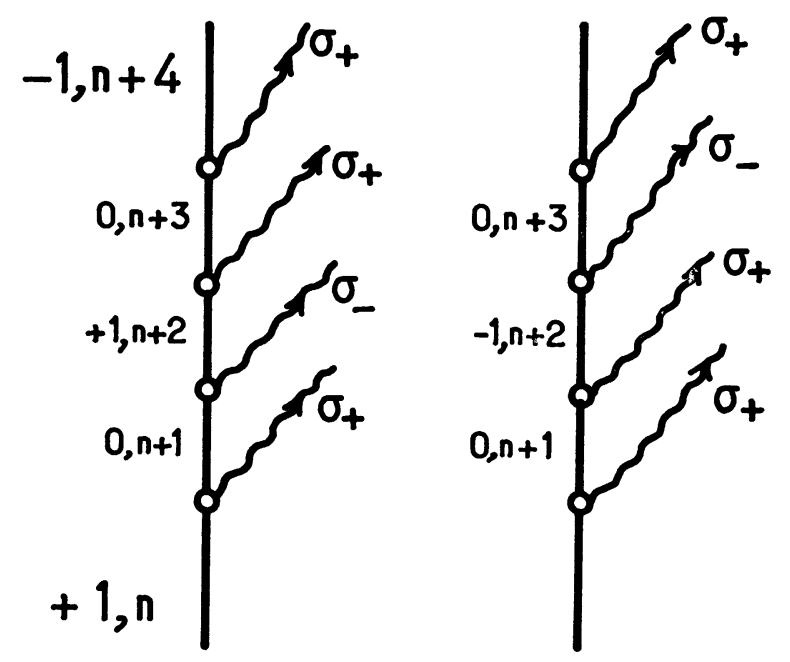

FIG. 6. - Schématisation des deux amplitudes de diffusion permettant de relier l'état $|+1, n\rangle$ à l'état $|-1, n+4\rangle$. Pour $\omega_{0}=2 \omega$, ces deux amplitudes interfèrent destructivement.

couplage possibles. Cette interférence destructive, que l'on observe sur un grand nombre de croisements quelle que soit la taille du spin ne peut être accidentelle et doit répondre à une raison profonde. En fait, comme il a déjà été montré dans la référence [2], l'existence de ces croisements nouveaux est une conséquence du théorème important de Majorana [12] qui relie l'évolution d'un spin $J>\frac{1}{2}$ dans un champ magnétique à celle d'un spin $J=\frac{1}{2}$ de même rapport gyromagnétique plongé dans le même champ. De façon plus précise, il est possible de construire à l'aide d'une règle géométrique simple les diagrammes d'énergie d'un spin $J$ habillé quelconque à partir de ceux d'un spin $J=\frac{1}{2}$. Cette règle, qui constitue en quelque sorte une correspondance quantique du théorème de Majorana, permet de prévoir d'emblée la position de tous les croisements de niveaux dans le diagramme d'énergie d'un spin $J$ habillé. Nous rappelons au paragraphe suivant cette formulation quantique du théorème de Majorana dont la démonstration complète peut être trouvée dans la référence [2], et qui joue un rôle essentiel dans l'étude des croisements de niveaux de l'atome habillé.

3.2 Formulation QUANTIQUe DU THÉORÈME DE MAJORANA ET DÉTERMINATION DU DIAGRAMME D'ÉNERGIE D'UN SPIN $J>\frac{1}{2}$ HABILLÉ. - 3.2.1 Définition des multiplicités de niveaux de l'atome habillé. - Il est commode pour l'étude des niveaux de l'atome habillé dans le cas $J>\frac{1}{2}$ de définir des multiplicités ou fuseaux de niveaux d'énergie. Conformément à la définition donnée dans [2], on appelle multiplicité $\left(\xi_{n}\right)$ dans un champ magnétique $H_{0}$ l'ensemble des $(2 J+1)$ niveaux $|\overline{J m n}\rangle$ qui se déduisent des états $|J m n\rangle$ de $n$ donné par la transformation adiabatique suivante :

$\alpha$ ) Le champ magnétique statique ayant une valeur $H_{\varepsilon}$ donnée très petite, on branche adiabatiquement la constante de couplage $\lambda$ entre l'atome et le champ. $\mathrm{Au}$ cours de cette opération, chaque état non per- 
turbé $|J m n\rangle$ se transforme continûment en un état de l'atome habillé dans le champ $H_{\varepsilon}$ que nous appellerons $\mid \overline{J m n}>_{\epsilon}$.

$\beta$ ) Le couplage une fois branché, on balaie adiabatiquement le champ statique depuis la valeur $H_{\varepsilon}$ jusqu'à la valeur $H_{0}$; partant du niveau $\mid \overline{J m n}>_{\varepsilon}$, on suit continûment au cours de ce balayage un niveau propre de l'atome habillé, pour parvenir à la fin de l'opération dans l'état $|\overline{J m n}\rangle$. Une telle transformation adiabatique suppose que l'on peut traverser les différents croisements rencontrés par le niveau $|\overline{J m n}\rangle$ lorsque $H_{0}$ varie, sans que le système subisse de transitions vers les autres niveaux. Nous avons vu dans [1], [2] que cette traversée adiabatique est toujours réalisée pour un croisement de première espèce et reste possible pour un croisement de deuxième espèce d'ordre quelconque à condition que le passage au point de croisement s'effectue suffisamment lentement. Appelant $U_{\mathrm{A}}$ la transformation unitaire adiabatique ainsi définie, on déduit les niveaux $\mid \overline{J m n}>$ des états non perturbés $\mid J m n>$ par la relation :

$$
|\overline{J m n}\rangle=U_{\mathrm{A}}|J m n\rangle \text {. }
$$

Notons que l'opérateur unitaire $U_{\mathrm{A}}$ commute avec toutes les opérations de symétrie éventuelles du hamiltonien, indépendantes de $\omega_{0}$. Il en résulte que les états $\mid \overline{J m n}>$ ont les mêmes nombres quantiques de symétrie que les états $|J m n\rangle$ dont ils sont issus. Par exemple, dans le cas d'un champ en polarisation $\sigma$, $\mid \overline{J m n}>$ est état propre de l'opérateur réflexion $S$ avec la valeur propre $(-1)^{J-m+n}$.

\subsubsection{Energie des niveaux dans une multiplicité de} l'atome habillé. - Sur les diagrammes de la figure 1 relatifs à un spin $\frac{1}{2}$, nous avons représenté en traits renforcés la multiplicité constituée des deux niveaux $\left|\overline{+\frac{1}{2}, n}\right\rangle$ et $\left|\overline{-\frac{1}{2}, n}\right\rangle$. Il apparaît clairement, dans tous les cas de polarisation possibles, que les deux niveaux sont symétriques par rapport à la droite d'ordonnée $n \omega$, et que l'on peut par conséquent mettre leur énergie sous la forme :

$$
E_{\overline{+1 / 2, n}}=n \omega \pm \frac{1}{2} \bar{\omega}_{0}
$$

où $\bar{\omega}_{0}$ représente la distance qui sépare les deux niveaux de la multiplicité et est naturellement une fonction de $H_{0}$, de $n$ et de la polarisation $\varepsilon$ de la radiofréquence. Il est possible d'interpréter la quantité $\pm \bar{\omega}_{0} / 2$ comme une énergie magnétique acquise par le spin dans l'état $\pm \frac{1}{2}$ lorsque, suivant la transformation $U_{\mathrm{A}}$, il est plongé, adiabatiquement et successivement, dans un champ RF de polarisation $\varepsilon$ contenant $n$ photons, et dans un champ statique $\mathbf{H}_{0}$.

La formulation quantique du théorème de Majorana [2] établit que, lorsque l'on soumet un spin $J$ quelconque, initialement dans l'état Zeeman $|J m\rangle$, à la transformation adiabatique $U_{\mathrm{A}}$, l'énergie magnétique qu'il acquiert en présence de $n$ photons est précisément égale à $m \bar{\omega}_{0}, \bar{\omega}_{0}$ étant la quantité définie plus haut et relative à un spin $\frac{1}{2}$ de même rapport gyromagnétique soumis à la même transformation $U_{\mathrm{A}}$. En conséquence, l'énergie du niveau $|\overline{J m n}\rangle$ de l'atome habillé doit s'écrire, quel que soit $J$ :

$$
E_{\overline{J m n}}=n \omega+m \bar{\omega}_{0}
$$

$\bar{\omega}_{0}$ étant une quantité indépendante de la taille du spin.

Pour démontrer cette propriété importante, on est amené à considérer que le système atomique de spin $J$ étudié est constitué par une assemblée totalement symétrique de $2 J$ spin $\frac{1}{2}$ identiques interagissant avec le champ de radiofréquence. On peut alors montrer qu'il est équivalent d'appliquer la transformation $U_{\mathrm{A}}$ globalement au spin $J$, ou successivement à chacun des $2 J$ spins $\frac{1}{2}$ qui le constituent. En établissant le bilan de l'énergie acquise par les $2 J$ spins lorsqu'on les introduit l'un après l'autre dans le champ de radiofréquence et dans le champ statique, on montre sans peine qu'un état $|\overline{J m n}\rangle$ doit avoir l'énergie donnée par la formule (3.3).

On déduit alors de ce résultat la règle essentielle suivante qui permet de construire immédiatement les diagrammes d'énergie relatifs à un spin $J$ de taille quelconque.

La branche $m$ de la multiplicité $\left(\mathcal{E}^{n}\right)$ relative au diagramme d'un spin $J$ habillé s'obtient par une affinité verticale de rapport $2 m$ sur la branche $m=+\frac{1}{2}$ de la multiplicité $\left(\mathcal{E}_{n}\right)$ relative à un spin $\frac{1}{2}$ habillé dans les mêmes conditions

C'est cette règle qui nous a permis de tracer à partir de la figure 1, l'ensemble des diagrammes d'énergie relatifs à un spin 1 qui sont reportés sur la figure 2 . Ces diagrammes permettent en fait d'étudier l'évolution d'un spin $J=1$ à partir de la connaissance de celle d'un spin $\frac{1}{2}$ plongé dans les mêmes champs statique et de radiofréquence. On peut donc bien dire que la règle mentionnée plus haut correspond à un théorème de Majorana quantique, dans la mesure où les formules classiques établies par Majorana [12] permettent de ramener le calcul des probabilités de transition d'un spin $J$ dans un champ de radiofréquence classique dépendant du temps à celui de la probabilité de transition entre les états $+\frac{1}{2}$ et $-\frac{1}{2}$ d'un spin $\frac{1}{2}$ soumis au même champ RF.

Nous allons étudier à présent les conséquences importantes de ce théorème, concernant les anticroisements de niveaux de l'atome habillé.

\subsection{ANTICROISEMENTS ET CROISEMENTS DE NIVEAUX} EN RELATION AVEC LE THÉORÈME DE MAJORANA. Un anticroisement de niveaux dans le diagramme d'énergie de l'atome habillé se manifeste toujours par un extrémum, lorsque l'on varie $\omega_{0}$, de l'énergie des niveaux qui s'anticroisent. D'après la formule (3.3), cet extrémum doit toujours correspondre à un maximum (ou à un minimum) de la fonction $\bar{\omega}_{0}\left(\omega_{0}\right)$. Or, cette fonction est indépendante de la taille du spin. Il s'ensuit que les anticroisements apparaissent dans le même champ magnétique statique, quel que soit 
la taille $J$ du spin, et qu'il ne peut donc y avoir d'anticroisement pour un spin $J$ donné que s'il existe déjà un anticroisement pour la même valeur de $\omega_{0}$ sur le diagramme correspondant du spin $\frac{1}{2}$. Comme conséquence très importante de cette propriété, les différents effets résonnants liés aux anticroisements (résonance magnétique à un ou plusieurs quanta) ne peuvent se manifester pour un spin quelconque que dans les champs magnétiques où ils ont lieu pour un spin $\frac{1}{2}$ de même rapport gyromagnétique. On retrouve ainsi un résultat bien connu qui apparaît également comme une conséquence des formules de Majorana classiques.

Un corollaire important de ce résultat est le suivant : lorsque pour un spin $J>\frac{1}{2}$ quelconque, deux ou plusieurs niveaux de $\mathscr{H}_{0}$ se croisent pour une valeur du champ magnétique où il n'existe pas d'anticroisement sur un spin $\frac{1}{2}$, ces niveaux doivent continuer à se croiser en présence du couplage $V$ entre l'atome et la radiofréquence. Si ce croisement est imposé par une règle de sélection, il sera de première espèce. $\mathrm{Si}$ par contre aucune règle de sélection basée sur des considérations de symétrie n'empêche deux des niveaux d'être reliés entre eux par $V$, alors le couplage correspondant doit nécessairement s'annuler sous l'effet d'une interférence destructive entre plusieurs processus, et le croisement de niveaux obtenu doit être de deuxième espèce. On l'appellera croisement de Majorana puisque son existence découle directement de l'application du théorème de Majorana à la construction du diagramme d'énergie de l'atome habillé.

Nous avons déjà donné au paragraphe 3.1 deux exemples de tels croisements de Majorana. Ils peuvent se produire lorsque deux niveaux sans symétrie définie se croisent pour une valeur non entière du champ magnétique, et correspondent alors à des croisements nouveaux n'existant pas pour un spin $\frac{1}{2}$ (cas 3.1.1). Ils peuvent également correspondre à un croisement entre niveaux de même symétrie pour une valeur du champ où il n'existe sur le spin $\frac{1}{2}$ qu'un croisement de première espèce (cas 3.1.2). Dans ce dernier cas, l'augmentation de la taille du spin conduit à une augmentation du nombre de niveaux qui se croisent dans le champ considéré, avec apparition de plusieurs niveaux ayant la même symétrie ; ces niveaux ne peuvent pourtant se repousser, puisque sur le spin $\frac{1}{2}$ il existe un croisement de niveaux pour cette valeur du champ; on a donc nécessairement apparition d'un croisement de Majorana de deuxième espèce. (C'est le cas de tous les croisements $\omega_{0}=2 p \omega$ dans le diagramme d'énergie d'un spin $J>\frac{1}{2}$ en présence d'un champ de RF en polarisation $\sigma$.) Enfin mention particulière doit être faite du croisement de niveaux en champ nul pour un champ RF en polarisation linéaire cohérente (ni $\sigma$, ni $\pi$ ). Ce croisement est, pour un spin $\frac{1}{2}$ (Fig. $1 d$ ), imposé par le théorème de Kramers comme conséquence de la symétrie par renversement du temps du système en champ nul (voir $\S 2.2$ ). Pour un spin $J>\frac{1}{2}$, cette symétrie n'impose plus une dégénérescence des $(2 J+1)$ sous niveaux de chaque multi- plicité de l'atome habillé. Il résulte cependant de la relation (3.2) que l'on a $\bar{\omega}_{0}=0$ pour $\omega_{0}=0$; en conséquence, d'après (3.3), les $(2 J+1)$ niveaux de chaque multiplicité se croisent effectivement en champ nul quel que soit $J$. C'est donc bien le théorème de Majorana qui impose l'existence de ce croisement pour $J>\frac{1}{2}$ dès lors qu'il existe pour $J=\frac{1}{2}$. En ce sens, nous dirons là encore qu'il s'agit d'un croisement de Majorana.

3.4 Ordre des Croisements de Majorana. Nous montrons en Appendice III que deux niveaux quelconques $\mid \overline{J m n}>$ et $\left|\overline{J m^{\prime} n^{\prime}}\right\rangle\left(m \neq m^{\prime}\right)$ de l'atome habillé sont d'ordre relatif $\left|m-m^{\prime}\right|-1$, à moins que ces deux niveaux ne soient de symétrie différente, auquel cas leur ordre relatif devient infini.

On en déduit que le croisement de deux niveaux $\mid \overline{J m n}>$ et $\left|\overline{J m^{\prime} n^{\prime}}\right\rangle$ est d'ordre $\left|m-m^{\prime}\right|-1$, sauf si une règle de sélection impose au croisement de devenir de première espèce.

Si plus de deux niveaux se croisent dans un champ donné, on appellera ordre du croisement l'ordre minimum relatif à tous les couples de niveaux que l'on peut envisager. Par exemple, dans le champ $\omega_{0}=2 \omega$ et pour une polarisation $\sigma$ de la radiofréquence, se croisent, dans le cas $J=1$, les trois niveaux $|\overline{1,0, n}\rangle$, $|\overline{1,1, n}\rangle$ et $|\overline{1,-1, n}\rangle$. Les deuxième et troisième niveaux sont de même symétrie, différente de celle du premier (cette symétrie est déterminée par la partie de $J-m+n$, cf. $\S 3.1)$. L'ordre relatif des niveaux $|\overline{1,1, n}\rangle$ et $|\overline{1,-1, n}\rangle$ qui ne peut donc être infini est égal à $\left|m-m^{\prime}\right|-1=1$. Par contre, l'ordre relatif de l'un de ces deux niveaux avec $|\overline{1,0, n}\rangle$ est infini. L'ordre du croisement est donc égal à 1 .

Nous avons récapitulé sur les deux tableaux cidessous tous les croisements de niveaux d'un spin $J=\frac{1}{2}$ et d'un spin $J=1$ dans les différents cas de polarisation possibles de la radiofréquence, en indiquant pour chaque croisement son espèce et son ordre. Sur une entrée du tableau, nous avons porté les polarisations, sur l'autre la position des différents croisements de $\mathscr{H}_{0}$. Lorsque le croisement devient un anticroisement sous l'effet du couplage, nous mettons la lettre A dans la case correspondante, si nous avons en présence du couplage un croisement de première espèce, nous mettrons la lettre $C_{1}$. S'il s'agit d'un croisement de deuxième espèce d'ordre $k$, nous le désignons par $\mathrm{C}_{2}(k)$.

Sur le diagramme du spin $\frac{1}{2}$, on constate que seuls apparaissent des croisements de première espèce et de deuxième espèce d'ordre zéro. Sur les diagrammes $\mathrm{du}$ spin 1, on observe également des croisements de deuxième espèce d'ordre 1 . Avec des spins de taille supérieure, on verrait apparaître dans les diagrammes des croisements de deuxième espèce d'ordre $\left|m-m^{\prime}\right|-1$ pouvant prendre une valeur quelconque supérieure à 1 . 


\begin{tabular}{|c|c|c|c|c|c|}
\hline \multirow[b]{2}{*}{$\begin{array}{l}\text { Position du croisement } \\
\text { ou de l'anticroisement }\end{array}$} & \multicolumn{5}{|c|}{$\operatorname{Spin} J=\frac{1}{2}$} \\
\hline & $\begin{array}{c}\text { Polarisation } \\
\pi\end{array}$ & $\sigma_{+}$ & $\sigma$ & $\begin{array}{l}\text { Polarisation } \\
\text { cohérente } \\
\text { linéaire }\end{array}$ & $\begin{array}{l}\text { Polarisation } \\
\text { cohérente } \\
\text { elliptique } \\
\text { ou circulaire }\end{array}$ \\
\hline$\omega_{0}=0^{-}$ & $\overline{\mathrm{C}_{1}}$ & $\overline{\mathrm{C}_{1}}$ & $\overline{\mathrm{C}_{1}}$ & $\overline{\mathrm{C}_{2}(0)}$ & $\overline{\mathrm{A}}$ \\
\hline$\omega_{0}=\omega$ & $\mathrm{C}_{1}$ & A & A & A & A \\
\hline $\begin{array}{c}\omega_{0}=(2 p+1) \omega \\
(p \neq 0)\end{array}$ & $\mathrm{C}_{1}$ & $\mathrm{C}_{1}$ & A & A & A \\
\hline \multirow[t]{3}{*}{$\begin{aligned} \omega_{0}= & (2 p) \omega \\
& (p \neq 0)\end{aligned}$} & $\mathrm{C}_{1}$ & $\mathrm{C}_{1}$ & $\mathrm{C}_{1}$ & A & A \\
\hline & \multicolumn{5}{|c|}{$\operatorname{Spin} J=1$} \\
\hline & $\pi$ & $\sigma$ & $\sigma$ & $\begin{array}{l}\text { Polarisation } \\
\text { cohérente } \\
\text { linéaire }\end{array}$ & $\begin{array}{l}\text { Polarisation } \\
\text { cohérente } \\
\text { elliptique } \\
\text { ou circulaire }\end{array}$ \\
\hline$\omega_{0}=0$ & $\mathrm{C}_{1}$ & $\mathrm{C}_{1}$ & $\mathrm{C}_{2}(1)$ & $\mathrm{C}_{2}(0)$ & A \\
\hline$\omega_{0}=\omega$ & $\mathrm{C}_{1}$ & A & A & A & A \\
\hline $\begin{aligned} \omega_{0}= & (2 p+1) \omega \\
& (p \neq 0)\end{aligned}$ & $\mathrm{C}_{1}$ & $\mathrm{C}_{1}$ & A & A & A \\
\hline $\begin{array}{c}\omega_{0}=2 p \omega \\
\quad(p \neq 0)\end{array}$ & $\mathrm{C}_{1}$ & $\mathrm{C}_{1}$ & $\mathrm{C}_{2}(1)$ & A & A \\
\hline$\omega_{0}=(2 p+1) \frac{\omega}{2}$ & $\mathrm{C}_{1}$ & $\mathrm{C}_{1}$ & $\mathrm{C}_{1}$ & $\mathrm{C}_{2}(1)$ & $\mathrm{C}_{2}(1)$ \\
\hline
\end{tabular}

En conclusion, nous avons mis en évidence l'existence de croisements de niveaux de deuxième espèce de tous ordres dans les diagrammes d'énergie de l'atome habillé, auxquels il est possible d'associer des résonances magnétiques d'un genre nouveau, dont les propriétés sont intermédiaires entre celles des résonances à plusieurs quanta et celles des résonances de cohérence ordinaires. Nous donnons au paragraphe suivant une étude expérimentale de ces résonances nouvelles. Nous les avons interprétées ici à l'aide d'une analyse globale du diagramme d'énergie de l'atome habillé. Il peut être intéressant de les comprendre également en termes de processus élémentaires d'absorption et d'émission de photons en adoptant le point de vue des diagrammes de Feynman développé dans la référence [3] pour interpréter les résonances de cohérence ordinaires associées aux croisements de première espèce. Cette interprétation diagrammatique, qui s'avère beaucoup plus délicate dans le cas des résonances associées aux croisements de deuxième espèce, est esquissée en Appendice IV.

4. Etude expérimentale d'une résonance à un croisement de Majorana. - Les résonances correspondant aux différents croisements de deuxième espèce d'un atome habillé ne sont pas toutes observables. Nous allons indiquer maintenant celles qui sont susceptibles d'être détectées optiquement, et décrire une expérience mettant en évidence l'une d'entre elles.
4.1 CONDITION D'OBSERVATION D'UNE RÉSONANCE EN POMPAGE LONGITUDINAL, A UN CROISEMENT DE DEUXIÈME ESPÈCE. - Dans la référence [1], nous avons montré que la résonance associée à un croisement de deuxième espèce pouvait être observée en utilisant une excitation longitudinale des états non perturbés. Une telle excitation peut en effet introduire une cohérence entre les états perturbés : la variation résonnante de cette cohérence au point de croisement est responsable de l'apparition de la résonance. Pour l'observer, il faut donc exciter convenablement les populations du système, afin qu'apparaisse une cohérence entre les niveaux perturbés qui se croisent.

Dans le cas de l'atome habillé, ce sont des observables atomiques qui sont préparées et détectées (par pompage optique et détection de la lumière absorbée ou diffusée). Les processus de préparation et de détection sont courts comparés au temps d'évolution dû au couplage avec le champ de radiofréquence. Immédiatement après l'excitation, la matrice densité du système total est le produit tensoriel d'une matrice densité atomique $\sigma^{(e x c)}$ et de la matrice densité du champ de radiofréquence $\rho_{\mathrm{RF}}$ [2]. L'excitation étant longitudinale, $\sigma^{(\mathbf{e x c})}$ est diagonale dans la base des états propres de $J_{z}$. On distingue différents types de préparation, suivant qu'on introduit :

$$
\begin{aligned}
& \text { - de l'orientation, si } \\
& \qquad \sigma^{(\mathrm{exc})}=a+b J_{\mathrm{z}}
\end{aligned}
$$

$a$ et $b$ étant des constantes, 
- de l'alignement, si

$$
\sigma^{(\mathrm{exc})}=c+d J_{\mathrm{z}}^{2}
$$

- des observables d'ordre tensoriel $p$, si $\sigma^{(\text {exc) }}$ s'écrit en fonction de $J_{z}$ comme un polynôme de degré $p$.

Pour simplifier les calculs, nous supposerons le champ de RF incohérent et caractérisé par une matrice densité $\rho_{\mathrm{RF}}$ diagonale dans la base $|n\rangle$, la distribution des populations étant une distribution de Poisson assez large pour qu'on puisse prendre $\rho_{\mathrm{RF}} \approx 1$ [2].

Pour pouvoir observer la résonance associée à un croisement de Majorana entre les niveaux $|\overline{J m n}\rangle$ et $\mid \overline{J m^{\prime} n^{\prime}}>$, il faut que l'on ait :

$$
<\overline{J m n}\left|\sigma^{(\mathrm{exc})} \otimes \mathbf{1}_{\mathrm{RF}}\right| \overline{J m^{\prime} n^{\prime}}>\neq 0 \text {. }
$$

Pour discuter la valeur de cet élément de matrice, nous allons utiliser la relation suivante, démontrée en Appendice II

$$
\begin{gathered}
\left\langle\overline{J m_{1} n_{1}}\left|J_{\mathrm{z}}\right| \overline{J m_{2} n_{2}}\right\rangle= \\
=\left\langle\overline{J m_{1} n_{1}} \mid \frac{\mathrm{d}}{\mathrm{d} \omega_{0}} \overline{J m_{2} n_{2}}\right\rangle\left(E_{\overline{m_{2} n_{2}}}-E_{\overline{m_{1} n_{1}}}\right) .
\end{gathered}
$$

Elle montre que $J_{z}$ n'a d'élément de matrice qu'entre des niveaux d'ordre relatif zéro. De façon plus générale, considérons l'élément de matrice

$$
<\overline{J m n}\left|J_{z}^{p}\right| \overline{J m^{\prime} n^{\prime}}>\text {. }
$$

On introduit $(p-1)$ fois la relation de fermeture relative aux vecteurs propres de $\mathcal{H}$ et on utilise autant de fois la relation (4.2). L'expression (4.3) s'écrit alors sous la forme d'une somme de produits de facteurs du type $\left\langle\overline{J m_{\mathbf{i}} n_{\mathbf{i}}} \mid \frac{\mathrm{d}}{\mathrm{d} \omega_{0}} \overline{J m_{\mathbf{j}} n_{\mathbf{j}}}\right\rangle$ qui forment une chaîne entre $|\overline{J m n}\rangle$ et $\left|\overline{J m^{\prime} n^{\prime}}\right\rangle$ via $(p-1)$ états intermédiaires. L'élément de matrice de $J_{z}^{p}$ entre $\mid \overline{J m n}>$ et $\mid \overline{J m^{\prime} n^{\prime}}>$ ne peut donc être différent de zéro que si $(p-1)$ est supérieur ou égal à l'ordre relatif des deux niveaux (cet ordre représente en effet le nombre minimum d'états intermédiaires permettant de former une chaîne non nulle entre ces niveaux). Or nous avons vu au paragraphe 3.4 que l'ordre relatif de deux niveaux $|\overline{J m n}\rangle$ et $\left|\overline{J m^{\prime} n^{\prime}}\right\rangle$ qui ne sont pas de symétrie différente est égal à $\left|m-m^{\prime}\right|-1$. On aboutit donc à la conclusion suivante: pour observer une résonance en pompage optique longitudinale au croisement de ces deux niveaux, il faut que le pompage optique et la détection portent sur des observables tensorielles d'ordre au moins $\left|m-m^{\prime}\right|\left(^{1}\right)$.

(1) Les problèmes des conditions d'observation des résonances pour un spin quelconque habillé par un champ RF se traite de façon plus complète dans le formalisme des opérateurs tensoriels irréductibles (voir [2]).
Expérimentalement, nous avons choisi d'étudier la résonance que l'on peut observer dans le champ $\omega_{0}=3 \omega / 2$ sur un atome de spin supérieur ou égal à 1 , habillé par un champ de radiofréquence de polarisation linéaire quelconque.

4.2 PrinciPe DE L'EXPÉRIENCE. - Les expériences ont été réalisées sur des atomes de ${ }^{201} \mathrm{Hg}$ dans l'état fondamental qui a un $\operatorname{spin} J=\frac{3}{2}$. La figure 7 représente deux fuseaux du diagramme d'énergie de ce spin habillé par un champ RF linéaire lorsque l'angle $\alpha$ entre la direction $\mathrm{Oz}$ du champ $\mathrm{RF}$ et $\mathrm{Oz}$ du champ magnétique statique est différent de 0 et $\pi / 2$ (polarisation ni $\pi$, ni $\sigma$ ). Les niveaux $\left|\frac{3}{2}, n-1\right\rangle$ et $\left|\overline{-\frac{1}{2}, n}\right\rangle$ se croisent dans le champ $\omega_{0}=3 \omega / 2$ (de même que $\left|\overline{\frac{1}{2}, n-1}\right\rangle$ et $\mid \overline{\left.-\frac{3}{2}, n\right\rangle}$ ), formant un croisement de Majorana d'ordre 1 car $\left|m-m^{\prime}\right|=2$. Pour le détecter en pompage longitudinal, il faut par suite exciter et observer de l'alignement. Remarquons enfin que le croisement devient de première espèce si $\alpha=0$ ou $\pi / 2$ (champ de RF de polarisation $\pi$ ou $\sigma$ ), car les niveaux qui se croisent sont alors de symétrie différente.

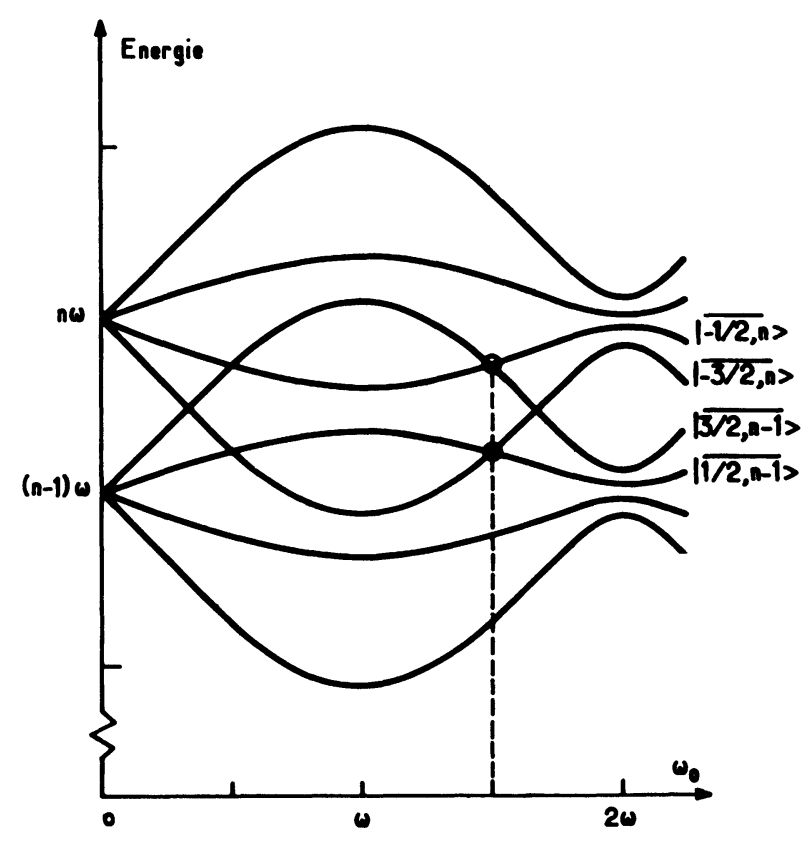

Fig. 7. - Diagramme d'énergie d'un spin $3 / 2$ habillé par des photons RF de polarisation linéaire cohérente (ni $\pi$, ni $\sigma$ ). Les croisements de niveaux de deuxième espèce $\omega_{0}=3 \omega / 2$ sont indiqués par des cercles.

Pour obtenir un alignement longitudinal, on utilise les techniques classiques du pompage optique [13] : la cellule de résonance contenant les atomes de ${ }^{201} \mathrm{Hg}$ est irradiée par le rayonnement à $2537 \AA$ issu d'une lampe à ${ }^{198} \mathrm{Hg}$, et polarisée dans la direction $\mathrm{Oz} \mathrm{du}$ champ statique (Fig. 8).

On peut montrer [2], [14] que la résonance ne se traduit pas seulement par une variation de l'intensité de la lumière de fluorescence : à cause du caractère cohérent du champ de RF, cette lumière est modulée 


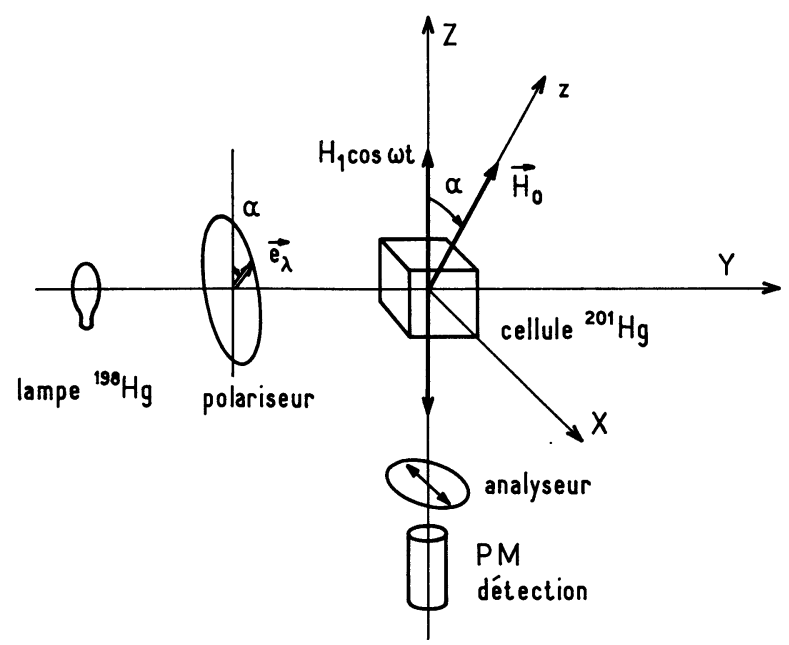

FIG. 8. - Montage expérimental utilisé pour observer les résonances correspondant aux croisements de niveaux de deuxième espèce de la figure 7 . On notera que le pompage optique est «longitudinal » $\left(\mathbf{e}_{\lambda} / /\right.$ à $\left.\mathbf{H}_{0}\right)$.

à la fréquence $\omega$ et ses harmoniques et la résonance se manifeste également sur l'amplitude de ces modulations.

La modulation $3 \omega$ subit en particulier une variation importante et elle a été utilisée pour détecter commodément la résonance. L'expression théorique du signal à l'ordre 3 en $\omega_{1} / \omega$ est calculée en détail dans la référence [14]. On trouve :

$$
L_{\mathrm{F}}(3 \omega)=K\left(\frac{\omega_{1}}{\omega}\right)^{3} \frac{\mathrm{e}^{-3 \mathrm{i} \omega t}}{\Gamma+\mathrm{i}\left(2 \omega_{0}-3 \omega\right)} \sin ^{2} \alpha \cos \alpha
$$

$K$ est une constante, $1 / \Gamma$ est le temps de relaxation de l'alignement (environ $1 \mathrm{~s}$ dans l'expérience décrite). Remarquons que grâce au facteur $\sin ^{2} \alpha \cos \alpha$, l'amplitude de la résonance s'annule lorsque le croisement devient de première espèce $(\alpha=0$ ou $\pi / 2)$.

4.3 Résultats expéRIMENTAux. - La figure 9 représente le spectre de résonances que l'on observe quand on balaie le champ statique $H_{0}$ de la valeur 0 à la

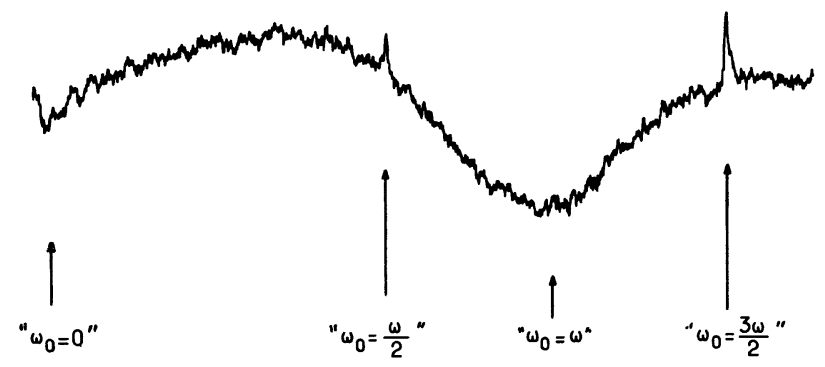

Fig. 9. - Spectre de résonances observé lorsqu'on balaie $H_{0}$ dans les conditions expérimentales de la figure 8 . Outre la résonance $\omega_{0}=3 \omega / 2$ cherchée, on observe la résonance d'anticroisement $\omega_{0}=\omega$ largement saturée et la résonance de croisement $\omega_{0}=\omega / 2$. Toutes ces résonances sont déplacées par rapport à leur position théorique en raison de l'intensité élevée de la radiofréquence $\left(\omega / 2 \pi=120 \mathrm{~Hz} ; \omega_{1} / \omega=1,25\right)$. valeur $2 \omega$. On observe la résonance d'anticroisement $\omega_{0}=\omega$ largement saturée et sur les bords deux résonances très fines qui correspondent aux croisements de deuxième espèce à $\omega_{0}=\omega / 2$ et $\omega_{0}=3 \omega / 2$ (en fait, ces résonances sont déplacées vis-à-vis de leur position extrapolée à couplage nul). Cette courbe correspond à une fréquence du champ oscillant de 120 cycles par seconde et un rapport $\omega_{1} / \omega$ égal à 1,25 .

Pour les expériences dont les résultats sont présentés ci-dessous, nous avons conservé la même fréquence pour le champ oscillant, mais nous avons pris $\omega_{1} / \omega$ égal à 0,7 de telle manière que notre expression théorique valable seulement au troisième ordre en $\omega_{1} / \omega$ puisse être utilisée.

Sur la figure 10, on a porté l'intensité de la résonance en fonction de l'angle $\alpha$. On vérifie que la résonance disparaît lorsque le croisement devient de

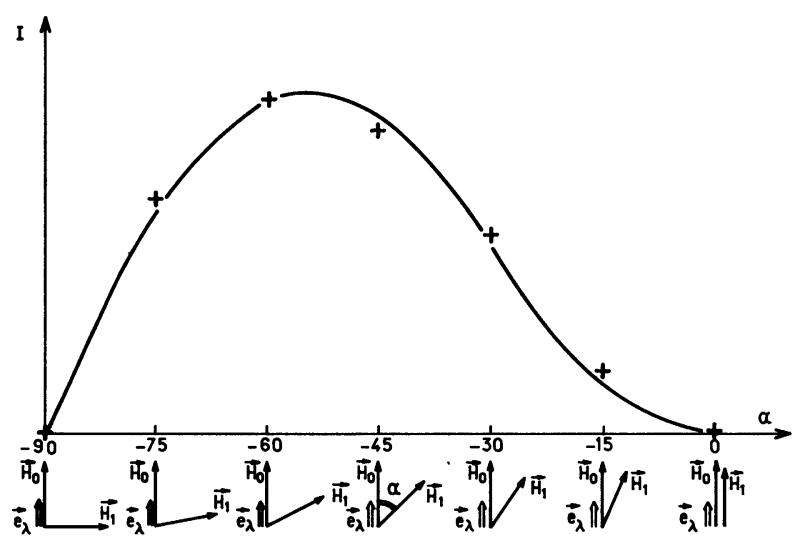

Fig. 10. - Variations de l'intensité de la résonance de croisement de deuxième espèce $\omega_{0}=3 \omega / 2$ en fonction de l'angle $\alpha$ entre $\mathbf{H}_{0}$ et $\mathbf{H}_{1}$. Le pompage est toujours longitudinal $\left(\mathbf{e}_{\lambda} / /\right.$ à $\left.\mathbf{H}_{0}\right)$. La courbe est théorique et les points expérimentaux.

première espèce ( $\alpha=0$ et $\alpha=\pi / 2$ ), et que les points expérimentaux sont conformes aux prévisions théoriques de la formule (4.4) représentées par la courbe en trait plein. Nous avons vérifié que l'existence de la résonance ne pouvait être attribuée à un défaut de parallélisme entre les polarisations lumineuses et le champ statique : en effet si l'angle $\beta$ entre $\mathbf{H}_{0}$ et le vecteur polarisation n'était pas tout à fait nul, le pompage optique introduirait directement une cohérence atomique et la résonance observée ne serait pas caractéristique d'un croisement de deuxième espèce. La figure 11 montre par exemple les variations de l'intensité $I$ de la résonance lorsqu'on fait varier $\beta$ autour de 0 . ( $\alpha$ est fixé à $-45^{\circ}$.) On n'observe aucune variation relative importante de l'intensité de la résonance. On peut donc traverser la valeur $\beta=0$, c'est-à-dire passer d'un pompage optique transversal à un pompage longitudinal, sans annulation de l'intensité de la résonance. Ceci confirme d'ailleurs que pour un croisement de deuxième espèce il n'y a pas physiquement de différence de nature entre les résonances en pompage longitudinal et transversal. Par contre, 


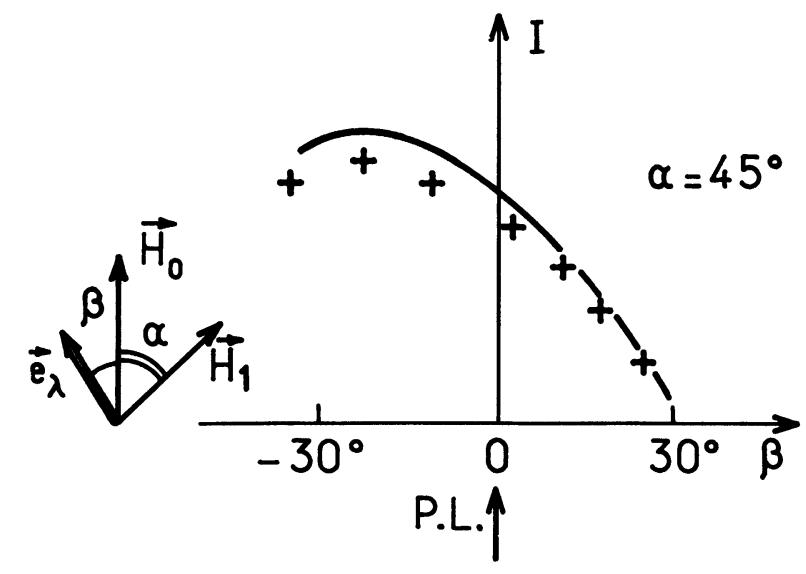

FIG. 11. - Variations de l'intensité de la résonance en fonction du caractère longitudinal ou transversal du pompage. Pour $\alpha=45^{\circ}$, le croisement $\omega_{0}=3 \omega / 2$ est de deuxième espèce et la résonance apparaît aussi bien en pompage longitudinal $(\beta=0)$ qu'en pompage transversal $(\beta \neq 0)$.

la figure 12 montre que, lorsque $\alpha=0$ ou $\pi / 2$, c'est-àdire lorsque le croisement est de première espèce, la résonance disparaît en pompage longitudinal, mettant ainsi bien en évidence la différence essentielle entre croisement de première et deuxième espèce.

Enfin, bien qu'une étude systématique n'ait pu être faite en raison du rapport signal/bruit insuffisant, il apparaît clairement que cette résonance ne subit pas un élargissement de radiofréquence. Lorsqu'on fait varier $\omega_{1} / \omega$ jusqu'à 1 , la largeur est toujours du même
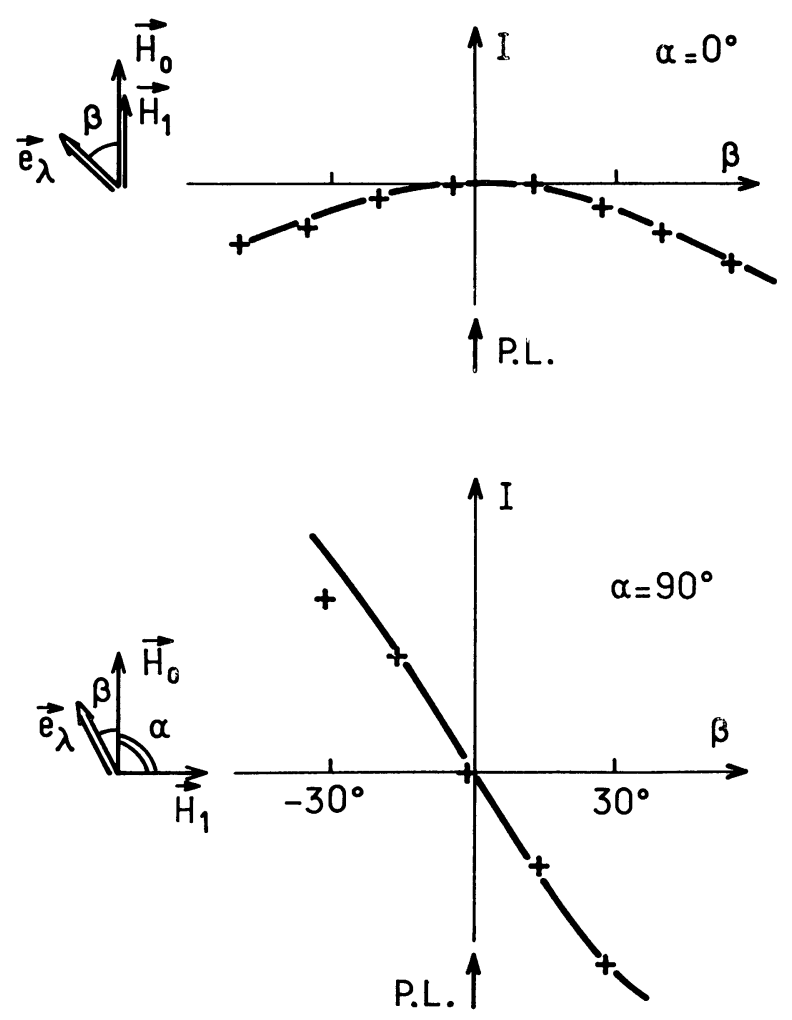

Fig. 12. - Variations de l'intensité de la résonance en fonction du caractère longitudinal ou transversal du pompage. Pour $\alpha=0$ ou $\alpha=90^{\circ}$, le croisement $\omega_{0}=3 \omega / 2$ est de première espèce et la résonance disparaît en pompage longitudinal $(\beta=0)$. ordre de grandeur que celle de la résonance Hanle, déterminée par le temps de relaxation $1 / \Gamma$. On voit d'ailleurs sur la figure 9 que, même pour $\omega_{1} / \omega \sim 1,25$, la largeur de la résonance reste de l'ordre de quelques hertz.

En conclusion, l'expérience confirme l'existence d'une résonance au voisinage du point de croisement de deuxième espèce $\omega_{0}=3 \omega / 2$. Conformément aux prévisions théoriques, cette résonance peut être observée en pompage longitudinal. Sa largeur est déterminée par le temps de relaxation atomique.

Une autre résonance de Majorana a été étudiée en détail : il s'agit de la résonance $\omega_{0}=0$ pour une polarisation $\sigma$ du champ de radiofréquence. La théorie et les expériences correspondantes sont présentées dans une autre publication [15].

Appendice I : Opérateurs de symétrie de l'atome habillé. - Il s'agit de donner l'expression des opérateurs qui décrivent la symétrie par rapport au plan xOy $(S)$ et le renversement du temps $(T)$ pour un spin $J$ habillé par un champ de radiofréquence de polarisation linéaire, parallèle à $O x$.

1) Opérateurs de symétrie atomique. - Ils sont bien connus [16]. Pour un spin $J$, l'opérateur $S$ s'écrit

d'autre part,

$$
\begin{aligned}
& S=\mathrm{i}^{2 J} \mathrm{e}^{-\mathrm{i} \pi J z} \\
& T=\mathrm{e}^{-\mathrm{i} \pi J y} K_{0}
\end{aligned}
$$

où $K_{0}$ est l'opérateur conjugaison complexe dans la base standard associée à $\mathbf{J}^{2}, J_{z}$.

Les observables atomiques se transforment de la façon suivante :

$$
\begin{aligned}
S J_{\mathrm{z}} S^{+} & =J_{\mathrm{z}} ; \quad S J_{\mathrm{x}} S^{+}=-J_{\mathrm{x}} ; \quad S J_{\mathrm{y}} S^{+}=-J_{\mathrm{y}} \\
T \mathbf{J} T^{+} & =-\mathbf{J} .
\end{aligned}
$$

2) Opérateurs de transformation du Champ RF QUANTIFIÉ. - Le problème est un peu moins simple, dans la mesure où l'on doit considérer l'ensemble des modes du champ électromagnétique et non pas uniquement celui qui est rempli. On peut cependant dans certains cas choisir les modes propres servant à quantifier le champ, de façon que leur structure géométrique soit invariante dans les opérations de symétrie $\left({ }^{2}\right)$. On peut alors ignorer l'existence des modes vides, puisque les opérateurs du mode plein considéré sont transformés en des opérateurs du même mode. Pour décrire le champ RF de polarisation parallèle à $\mathrm{Ox}$, nous prendrons un mode stationnaire, de potentiel vecteur :

$$
\mathbf{A}=A_{0} \varepsilon_{z}\left(a+a^{+}\right) \sin k \mathrm{y} .
$$

(2) Remarquons qu'un tel choix exclut une polarisation circulaire, car elle n'est pas invariante par renversement du temps. 
Les opérateurs champ magnétique $\mathbf{H}_{1}$ et champ électrique $\mathbf{E}$ s'écrivent alors :

$$
\begin{aligned}
& \mathbf{H}_{1}=A_{0} \boldsymbol{\varepsilon}_{\mathrm{x}}\left(a+a^{+}\right) k \cos k \mathrm{y} \\
& \mathbf{E}=A_{0} \boldsymbol{\varepsilon}_{\mathrm{z}} \mathrm{i}\left(a-a^{+}\right) \omega \sin k \mathrm{y}
\end{aligned}
$$

$\boldsymbol{\varepsilon}_{\mathrm{x}}, \boldsymbol{\varepsilon}_{\mathrm{y}}, \boldsymbol{\varepsilon}_{\mathrm{z}}$ sont les vecteurs unitaires des 3 axes.

Pour ce choix particulier du mode décrivant le champ RF, $S$ et $T$ se déterminent simplement :

a) $S$ doit changer $\mathbf{H}_{1}$ en $-\mathbf{H}_{1}$ et $\mathbf{E}$ en $-\mathbf{E}$. Ces conditions sont réalisées si $\mathrm{SaS}^{+}=-a$ et on vérifie que

$$
S=\mathrm{e}^{+\mathrm{i} \pi a^{+} a}
$$

est une solution [il suffit de remarquer que, pour une fonction analytique $f$, on a la relation

$$
\left.a f\left(a^{+} a\right)=f\left(a^{+} a+1\right) a\right] \text {. }
$$

b) $T$ doit changer $\mathbf{H}_{1}$ en $-\mathbf{H}_{1}$ et garder $\mathbf{E}$ invariant : Par ailleurs, $T$ est antiunitaire et anticommute avec i. Il suffit donc que $T$ vérifie $T a T^{+}=-a$ pour que $T$ soit l'opérateur antiunitaire cherché. On en déduit que $T$ peut s'écrire sous la forme :

$$
T=\mathrm{e}^{+\mathrm{i} \pi a^{+} a} K_{0}
$$

où $K_{0}$ est l'opérateur conjugaison complexe dans la base $\{|n\rangle\} . K_{0}$ commute évidemment avec $a$ et $a^{+}$, puisque leurs éléments de matrice sont réels dans la base considérée.

Finalement, les opérateurs qui décrivent les opérations $S$ et $T$ pour l'atome habillé ont les expressions suivantes :

$$
\begin{aligned}
& S=\mathrm{i}^{2 J} \mathrm{e}^{-\mathrm{i} \pi\left(J_{z}-a^{+} a\right)} \\
& T=\mathrm{e}^{-\mathrm{i} \pi\left(J_{\mathbf{z}}-a^{+} a\right)} K_{0}
\end{aligned}
$$

$K_{0}$ étant la conjugaison complexe dans la base

$$
\left\{\mathbf{J}^{2}, J_{z}, a^{+} a\right\} \text {. }
$$

Appendice II : Etude de l'ordre relatif de deux niveaux quelconques d'un spin $\mathbf{J}=\frac{1}{2}$ habillé. - Nous avons déterminé au paragraphe 2.1 l'ordre relatif de deux niveaux quelconques d'un spin $J=\frac{1}{2}$ habillé dans le cas où le champ RF a une polarisation $\pi$ ou $\sigma_{+}$. Nous nous proposons dans cet Appendice d'étudier l'ordre des niveaux dans le cas plus compliqué où le champ RF a une polarisation cohérente quelconque (superposition des polarisations $\sigma_{+}, \sigma_{-}$et $\pi$ ).

Pour déterminer l'ordre de deux niveaux, on est amené à calculer des expressions de la forme

$$
\left\langle\bar{a} \mid \frac{\mathrm{d} \bar{b}}{\mathrm{~d} \omega_{0}}\right\rangle \text {. }
$$

Nous allons montrer que ces expressions sont en fait proportionnelles à l'élément de matrice de $J_{z}$ entre $|\bar{a}\rangle$ et $|\bar{b}\rangle$. En effet, on déduit immédiatement de l'expression (1.1) du hamiltonien de l'atome habillé la relation :

$$
\frac{\mathrm{d} \mathscr{H}}{\mathrm{d} \omega_{0}}=J_{\mathrm{z}}
$$

qui permet d'écrire, en dérivant la quantité identiquement nulle $<\overline{\mathrm{a}}|\mathfrak{H e}| \overline{\mathrm{b}}>$ :

$$
\begin{aligned}
\frac{\mathrm{d}}{\mathrm{d} \omega_{0}} & <\overline{\mathrm{a}}|\mathfrak{H}| \overline{\mathrm{b}}\rangle= \\
& =\left\langle\overline{\mathrm{a}}\left|J_{\mathrm{z}}\right| \overline{\mathrm{b}}\right\rangle+E_{\overline{\mathrm{b}}}\left\langle\frac{\mathrm{d} \overline{\mathrm{a}}}{\mathrm{d} \omega_{0}} \mid \overline{\mathrm{b}}\right\rangle+E_{\mathrm{a}}\left\langle\overline{\mathrm{a}} \mid \frac{\mathrm{d} \bar{b}}{\mathrm{~d} \omega_{0}}\right\rangle \\
& =0 .
\end{aligned}
$$

Enfin, si l'on tient compte de la relation :

$$
\frac{\mathrm{d}}{\mathrm{d} \omega_{0}}\langle\overline{\mathrm{a}} \mid \overline{\mathrm{b}}\rangle=\left\langle\overline{\mathrm{a}} \mid \frac{\mathrm{d} \overline{\mathrm{b}}}{\mathrm{d} \omega_{0}}\right\rangle+\left\langle\frac{\mathrm{d} \overline{\mathrm{a}}}{\mathrm{d} \omega_{0}} \mid \overline{\mathrm{b}}\right\rangle=0 \text {. }
$$

On déduit de (A.II.2), l'égalité importante :

$$
\left\langle\overline{\mathrm{a}}\left|J_{\mathrm{z}}\right| \overline{\mathrm{b}}\right\rangle=\left(E_{\mathrm{a}}^{-}-E_{\overline{\mathrm{b}}}\right)\left\langle\overline{\mathrm{a}} \mid \frac{\mathrm{d} \bar{b}}{\mathrm{~d} \omega_{0}}\right\rangle
$$

qui démontre le résultat annoncé. Ainsi, pour que deux niveaux $|\bar{a}\rangle$ et $|\bar{b}\rangle$ de l'atome habillé soient d'ordre relatif zéro, il suffit que la quantité $\left\langle\bar{a}\left|J_{z}\right| \bar{b}\right\rangle$ ne soit pas identiquement nulle.

Pour étudier l'ordre de deux niveaux quelconques $\mid \overline{m n}>$ et $\mid \overline{m^{\prime} n^{\prime}}>\left(m, m^{\prime}= \pm \frac{1}{2}\right)$, nous allons donc calculer les éléments de matrice $<\overline{m n}\left|J_{\mathrm{z}}\right| \overline{m^{\prime} n^{\prime}}>$. Nous effectuerons ce calcul au voisinage du champ statique nul et nous le limiterons au cas où la polarisation du champ habillant est linéaire, car nous connaissons alors, en champ $H_{0}$ nul, l'expression analytique exacte des fonctions d'onde de l'atome habillé, ce qui facilite considérablement le calcul de l'ordre relatif des niveaux. Nous verrons d'ailleurs qu'il est possible de généraliser aisément les résultats obtenus au cas où la polarisation de la RF est elliptique.

L'expression des états $|\overline{m n}\rangle$ pour un champ polarisé linéairement en champ $H_{0}$ faible, se déduit aisément des résultats de la référence [2]. Nous sup-

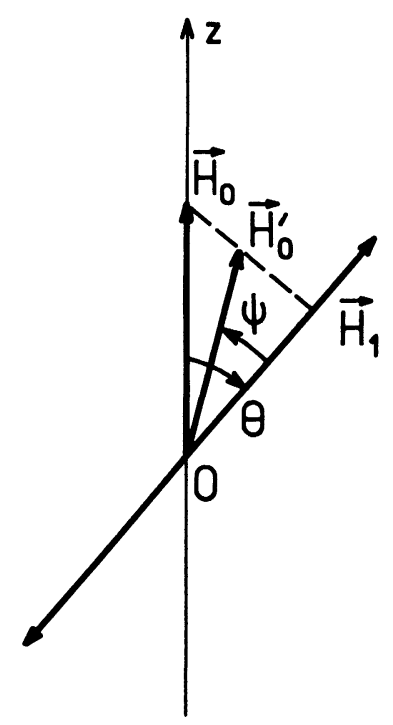

FIG. 13. 
poserons que $\mathbf{H}_{0}$ et $\mathbf{H}_{1}$ font un angle $\theta$ et nous alignerons comme nous l'avons fait dans tout cet article $\mathbf{H}_{0}$ le long de $\mathrm{Oz}$ (voir Fig. 13). Nous désignerons par $1 \pm>_{\mathrm{z}}$ les états de spin $+\frac{1}{2}$ et $-\frac{1}{2}$ le long de $\mathrm{Oz}$, par $\mid \pm>_{\theta}$ les états de spin $+\frac{1}{2}$ et $-\frac{1}{2}$ dans la direction de $\mathbf{H}_{1}, \mid \pm>_{\theta}$ se déduisant de $\mid \pm>_{z}$ par une rotation d'angle $\theta$ :

$$
\begin{aligned}
& \left|+>_{\theta}=\cos \frac{\theta}{2}\right|+>_{z}-\sin \frac{\theta}{2} \mid->_{z} \\
& \left|->_{\theta}=\sin \frac{\theta}{2}\right|+>_{z}+\cos \frac{\theta}{2} \mid->_{z} .
\end{aligned}
$$

Nous appellerons enfin, comme dans [2] $\left|\bar{n}_{ \pm}\right\rangle$les états propres déplacés du champ RF définis par:

$$
\mid \bar{n}_{ \pm}>=\exp \left( \pm \frac{\lambda}{2 \omega}\left(a-a^{+}\right) \mid n>\right.
$$

dont les produits scalaires vérifient les relations :

$$
\begin{aligned}
& <\bar{n}_{+}\left|\bar{n}_{-}^{\prime}\right\rangle=J_{n-n^{\prime}}\left(\frac{\omega_{1}}{\omega}\right) \\
& <\bar{n}_{ \pm}\left|\bar{n}_{ \pm}^{\prime}\right\rangle=\delta_{n n^{\prime}} .
\end{aligned}
$$

En champ $H_{0}$ nul, les états propres de l'atome habillé sont simplement les produits tensoriels définis par la relation

$$
\left| \pm \bar{n}>_{\theta}=\right| \pm>_{\theta} \mid \bar{n}_{ \pm}>
$$

En présence d'un petit champ $\mathbf{H}_{0}$, on déduit les niveaux d'énergie de l'atome habillé des niveaux calculés en champ nul en diagonalisant la perturbation $\omega_{0} J_{z}$ dans chaque multiplicité $\left| \pm>_{\theta}\right| \bar{n}_{ \pm}>$. Cette diagonalisation revient à effectuer une rotation fictive sur les états propres définis en champ nul, d'un angle $\psi$ donné par

$$
\operatorname{tg} \psi=J_{0}\left(\frac{\omega_{1}}{\omega}\right) \operatorname{tg} \theta
$$

L'action d'un petit champ statique dans chaque multiplicité de l'atome habillé est en effet équivalente à celle que produirait sur un spin $\frac{1}{2}$ libre un champ statique fictif $\mathbf{H}_{0}^{\prime}$ se déduisant de $\mathbf{H}_{0}$ par une affinité d'axe $\mathbf{H}_{1}$ et de rapport $J_{0}\left(\omega_{1} / \omega\right)$ (voir Fig. 13). On obtient ainsi les états propres de l'atome habillé, à l'ordre zéro en $\omega_{0}$, comme combinaison linéaire des états $|\overline{ \pm n}\rangle_{\theta}$ :

$$
\begin{aligned}
& \left|\overline{+n}>=\cos \frac{\psi}{2}\right| \overline{+n}>_{\theta}+\sin \frac{\psi}{2} \mid \overline{-n}>_{\theta} \\
& \left|\overline{-n}>=-\sin \frac{\psi}{2}\right| \overline{+n}>_{\theta}+\cos \frac{\psi}{2} \mid \overline{-n}>_{\theta} .
\end{aligned}
$$

Soit encore, compte tenu de (A.II.5) et (A.II.8) :

$$
\begin{aligned}
\mid \overline{+n}>= & \cos \frac{\psi}{2} \cos \frac{\theta}{2}\left|+>_{\mathrm{z}}\right| \bar{n}_{+}>+\sin \frac{\psi}{2} \sin \frac{\theta}{2}\left|+>_{\mathrm{z}}\right| \bar{n}_{-}>- \\
& \quad-\cos \frac{\psi}{2} \sin \frac{\theta}{2}\left|->_{\mathrm{z}}\right| \bar{n}_{+}>+\sin \frac{\psi}{2} \cos \frac{\theta}{2}\left|->_{\mathrm{z}}\right| \bar{n}_{-}> \\
\mid \overline{-n}>=- & \sin \frac{\psi}{2} \cos \frac{\theta}{2}\left|+>_{\mathrm{z}}\right| \bar{n}_{+}>+\cos \frac{\psi}{2} \sin \frac{\theta}{2}\left|+>_{\mathrm{z}}\right| \bar{n}_{-}>+ \\
& +\sin \frac{\psi}{2} \sin \frac{\theta}{2}\left|->_{\mathrm{z}}\right| \bar{n}_{+}>+\cos \frac{\psi}{2} \cos \frac{\theta}{2}\left|->_{\mathrm{z}}\right| \bar{n}_{-}>.
\end{aligned}
$$

Les relations (A.II.7) et (A.II.11) permettent alors de déterminer aisément les éléments de matrice de $J_{z}$ cherchés. On obtient notamment :

$$
\begin{aligned}
& <\overline{+n}\left|J_{\mathrm{z}}\right| \overline{+n^{\prime}}>=\frac{\cos \psi \cos \theta}{2} \delta_{n n^{\prime}}+\frac{\sin \psi \sin \theta}{2} J_{n-n^{\prime}}\left(\frac{\omega_{1}}{\omega}\right) \delta_{n-n^{\prime}, \text { pair }} \\
& \left\langle\overline{+n}\left|J_{\mathrm{z}}\right| \overline{-n^{\prime}}\right\rangle=-\frac{\cos \theta \sin \psi}{2} \delta_{n n^{\prime}}+\frac{\sin \theta \cos \psi}{2} J_{n-n^{\prime}}\left(\frac{\omega_{1}}{\omega}\right) \delta_{n-n^{\prime}, \text { pair }}+\frac{\sin \theta}{2} J_{n-n^{\prime}}\left(\frac{\omega_{1}}{\omega}\right) \delta_{n-n^{\prime}, \text { impair }} .
\end{aligned}
$$


Dans ces expressions, les symboles $\delta_{n-n^{\prime}}$, pair $\left(\delta_{n-n^{\prime}}\right.$, impair) sont des nombres égaux à 1 si $n-n^{\prime}$ est pair (impair), et à zéro si $n-n^{\prime}$ est impair (pair). On a utilisé pour établir les égalités A.II.12 et A.II.13 les relations de parité bien connues satisfaites par les fonctions de Bessel :

$$
J_{p}\left(\frac{\omega_{1}}{\omega}\right)=(-1)^{p} J_{-p}\left(\frac{\omega_{1}}{\omega}\right)=(-1)^{p} J_{p}\left(-\frac{\omega_{1}}{\omega}\right) .
$$

Nous sommes à présent en mesure de déterminer l'ordre relatif de deux niveaux de l'atome habillé pour un champ linéaire de polarisation quelconque : a) Champ RF en Pol.ARisation $\pi$. - On doit dans (A.II.12) et (A.II.13) faire $\theta=\psi=0$ ce qui donne

$$
<\overline{m n}\left|J_{\mathrm{z}}\right| \overline{m^{\prime} n^{\prime}}>=\frac{1}{2} \delta_{n n^{\prime}} \delta_{m m^{\prime}} \text {. }
$$

Cette relation reste d'ailleurs valable quel que soit le champ statique. On retrouve bien que deux niveaux distincts ne peuvent jamais être d'ordre zéro, c'est-àdire encore que tous les niveaux sont, dans ce cas, d'ordre relatif infini (cf. $\S 2.1$ ).

b) Cha.mp RF en polarisation $\sigma$. - On doit dans (A II.12) et (A.II.13) faire $\theta=\psi=\pi / 2$ ce qui donne

$$
\begin{gathered}
<\overline{+n}\left|J_{\mathrm{z}}\right| \overline{+n^{\prime}}>=\frac{1}{2} J_{n-n^{\prime}}\left(\frac{\omega_{1}}{\omega}\right) \delta_{n-n^{\prime}, \text { pair }}\left(n \neq n^{\prime}\right) \\
<\overline{+n}\left|J_{\mathrm{z}}\right| \overline{-n^{\prime}}>=\frac{1}{2} J_{n-n^{\prime}}\left(\frac{\omega_{1}}{\omega}\right) \delta_{n-n^{\prime}, \text { impair }}
\end{gathered}
$$

On déduit alors de (A.II.15) que si $n-n^{\prime}$ est pair les niveaux $\mid \overline{+n}>$ et $\mid \overline{+n^{\prime}}>$ sont d'ordre relatif zéro. Nous avons vu au paragraphe 2.1 que ces niveaux sont alors de même symétrie. Nous savons également que si $n-n^{\prime}$ est impair ces deux niveaux sont de symétries différentes et leur ordre relatif est alors infini. Il résulte d'autre part de (A.II.16) que les niveaux $\mid \overline{+n}>$ et $\left|\overline{-n^{\prime}}\right\rangle$ sont d'ordre relatif zéro si $n-n^{\prime}$ est impair (ces niveaux sont alors, comme nous l'avons vu au $\S 2.1$ de même symétrie). Si $n-n^{\prime}$ est pair au contraire, nous savons (cf. $\S 2.1$ ) que $\mid \overline{+n}>$ et $\mid \overline{-n^{\prime}}>$ sont de symétries différentes et leur ordre relatif est donc infini. Nous avons ainsi déterminé l'ordre relatif d'un couple quelconque des niveaux pour un champ RF en polarisation $\sigma$. Cet ordre est soit nul, soit infini suivant que les niveaux concernés ont ou non même symétrie.

c) Polarisation linéaire COHÉRente QuelconQue DE LA RF. $-\theta$ et $\psi$ sont alors différents de zéro et $\pi / 2$. On constate alors sans peine (A.II.13) que

$$
<\overline{+n}\left|J_{z}\right| \overline{-n^{\prime}}>
$$

est différent de zéro quelle que soit la parité de $n-n^{\prime}$ : deux niveaux $\mid \overline{+n}>$ et $\mid \overline{-n^{\prime}}>$ sont donc toujours d'ordre relatif zéro.

Par ailleurs on déduit de (A.II.12) les égalités :

$$
\begin{aligned}
\left\langle\overline{+n}\left|J_{\mathrm{z}}\right| \overline{+n^{\prime}}>\right. & =\frac{\sin \theta \sin \psi}{2} J_{n-n^{\prime}}\left(\frac{\omega_{1}}{\omega}\right) \text { si } n-n^{\prime} \text { est pair } \\
& =0 \text { si } n-n^{\prime} \text { est impair } .
\end{aligned}
$$

On peut donc dire que les deux niveaux parallèles $|\overline{+n}\rangle$ et $\mid \overline{+n^{\prime}}>$ sont d'ordre relatif zéro si $n-n^{\prime}$ est pair. Si $n-n^{\prime}$ est impair, on peut simplement constater que la quantité $\left\langle\overline{+n}\left|J_{z}\right| \overline{+n^{\prime}}\right\rangle$ est nulle à l'ordre zéro en $\omega_{0}$. En fait un calcul de perturbations des niveaux poussé à l'ordre suivant en $\omega_{0}$ permet de montrer que $\left\langle\overline{+n}\left|J_{z}\right| \overline{+n^{\prime}}>\right.$ n'est pas dans ce cas identiquement nul. On en déduit que deux niveaux quelconques $\mid \overline{+n}>$ et $\mid \overline{+n^{\prime}}>$ sont également d'ordre relatif zéro, quelle que soit la parité de $n-n^{\prime}$.

En conclusion, dans le cas d'une polarisation linéaire cohérente quelconque de la $R F$, deux niveaux arbitrairement choisis de l'atome habillé sont d'ordre relatif zéro.

Cette propriété ne dépend d'ailleurs pas du caractère linéaire du champ RF. Si le champ est de polarisation elliptique cohérente, on ne dispose généralement pas des fonctions d'onde exactes de l'atome habillé en champ nul, mais on peut toujours déterminer des fonctions d'onde approchées par un calcul de perturbation consistant à considérer le champ elliptique comme résultant de la composition de deux champs linéaires déphasés l'un par rapport à l'autre et polarisés dans des directions différentes, l'un de ces champs étant traité comme une perturbation vis-à-vis de l'autre. On montre aisément par un calcul de ce type que deux niveaux quelconques de l'atome habillé, sont comme pour un champ linéaire cohérent, d'ordre relatif zéro. Finalement tous les résultats relatifs à l'ordre des niveaux pour un spin $\frac{1}{2}$ habillé sont résumés dans le tableau suivant. 


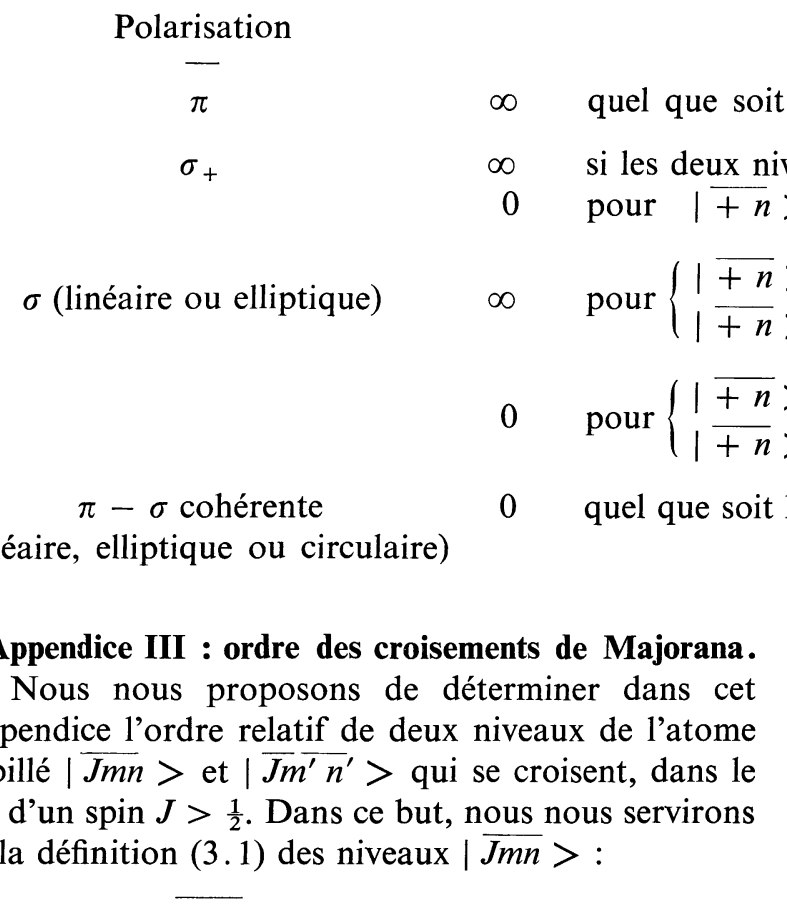

$$
\left|\overline{J m n}>=U_{\mathrm{A}}\right| J m n>\text {. }
$$

L'état non perturbé $\mid J m n>$ peut lui-même être décrit comme une superposition symétrique de $2 J$ spins $\frac{1}{2}$ en présence de $n$ photons de radiofréquence; $(J+m)$ spins sont dans l'état $+\frac{1}{2}$ et $(J-m)$ dans l'état $-\frac{1}{2}$. Nous écrirons donc :

$$
\left|J m n>=\alpha_{J m} S_{J m}\right|(+)_{J+m},(-)_{J-m}>\otimes \mid n>\text {. }
$$

Dans cette expression, $\mid(+)_{J+m},(-)_{J-m}>$ est le
Ordre relatif des niveaux

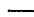

iveaux

$+n)$ différent

avec $n-n^{\prime}$ impair

avec $n-n^{\prime}$ pair

avec $n-n^{\prime}$ pair

avec $n-n^{\prime}$ impair

vecteur pour lequel les $J+m$ premiers spins sont dans l'état $+\frac{1}{2}$, les $J-m$ derniers dans l'état $-\frac{1}{2}$, $S_{J m}$ est l'opérateur de symétrisation.

$$
S_{J m}=\frac{1}{(2 J) !} \sum_{p} P
$$

ou $\sum_{p} P$ est la somme des $(2 J)$ ! permutations possibles de $2 J$ particules. Enfin $\alpha_{J m}$ est une constante de normalisation :

$$
\alpha_{J m}=\left[\frac{(2 J) !}{(J+m) !(J-m) !}\right]^{1 / 2} .
$$

Ces notations étant définies, étudions pour commencer le produit scalaire

$$
\left\langle\overline{J m n} \mid \frac{\mathrm{d}}{\mathrm{d} \omega_{0}} \overline{J m^{\prime} n^{\prime}}\right\rangle \text {. }
$$

Compte tenu de (3.1), nous pouvons écrire :

$$
\left\langle\overline{J m n} \mid \frac{\mathrm{d}}{\mathrm{d} \omega_{0}} \overline{J m^{\prime} n^{\prime}}\right\rangle=\left\langle J m n\left|U_{\mathrm{A}}^{+} \frac{\mathrm{d} U_{\mathrm{A}}}{\mathrm{d} \omega_{0}}\right| J m^{\prime} n^{\prime}\right\rangle .
$$

L'opération unitaire $U_{\mathrm{A}}$ peut être décomposée comme un produit commutatif [2] d'opérateurs agissant sur chacun des $2 J$ spins et décrivant le branchement successif sur chacun de ces spins de la radiofréquence et du champ statique :

$$
U_{\mathrm{A}}=U_{\mathrm{A}}(1) U_{\mathrm{A}}(2) \ldots U_{\mathrm{A}}(\mathrm{i}) \ldots U_{\mathrm{A}}(2 J) \text {. }
$$

Tenant compte du caractère commutatif des opérateurs unitaires $U_{\mathrm{A}}(\mathrm{i})$, on déduit sans peine de (A.III.5) la relation :

$$
U_{\mathrm{A}}^{+} \frac{\mathrm{d} U_{\mathrm{A}}}{\mathrm{d} \omega_{0}}=\sum_{\mathbf{i}} U_{\mathrm{A}}^{+}(\mathrm{i}) \frac{\mathrm{d} U_{\mathrm{A}}(\mathrm{i})}{\mathrm{d} \omega_{0}}
$$

qui permet de mettre (A.III.4) sous la forme

$$
\left\langle\overline{J m n} \mid \frac{\mathrm{d}}{\mathrm{d} \omega_{0}} \overline{J m^{\prime} n^{\prime}}\right\rangle=\left\langle J m n\left|\sum_{\mathrm{i}} U_{\mathrm{A}}^{+}(\mathrm{i}) \frac{\mathrm{d} U_{\mathrm{A}}(\mathrm{i})}{\mathrm{d} \omega_{0}}\right| J m^{\prime} n^{\prime}\right\rangle
$$

soit encore, compte tenu de (A.III.1) :

$$
\begin{aligned}
& \left\langle\overline{J m n} \mid \frac{\mathrm{d}}{\mathrm{d} \omega_{0}} \overline{J m^{\prime} n^{\prime}}\right\rangle= \\
& =\alpha_{J m} \alpha_{J m^{\prime}}\left\langle n\left|\otimes\left\langle(+)_{J+m},(-)_{J-m}\left|S_{J m}^{+} \sum_{\mathrm{i}} U_{\mathrm{A}}^{+}(\mathrm{i}) \frac{\mathrm{d} U_{\mathrm{A}}(\mathrm{i})}{\mathrm{d} \omega_{0}} S_{J m}\right|(+)_{J+m^{\prime}},(-)_{J-m^{\prime}}\right\rangle \otimes\right| n^{\prime}\right\rangle .
\end{aligned}
$$


$S_{J m}$ commutant avec l'opérateur symétrique $\sum_{\mathrm{i}} U_{\mathrm{A}}^{+}(\mathrm{i}) \frac{\mathrm{d} U_{\mathrm{A}}(\mathrm{i})}{\mathrm{d} \omega_{0}}$, on peut, en tenant compte de la relation $S_{J m} S_{J m}^{+}=S_{J m}$, simplifier (A.III.8) sous la forme :

$\left\langle\overline{J m n} \mid \frac{\mathrm{d}}{\mathrm{d} \omega_{0}} \overline{J m^{\prime} n^{\prime}}\right\rangle=\alpha_{J m} \alpha_{J m^{\prime}}\left\langle n\left|\otimes\left\langle(+)_{J+m},(-)_{J-m}\left|\sum_{\mathrm{i}} U_{\mathrm{A}}^{+}(\mathrm{i}) \frac{\mathrm{d} U_{\mathrm{A}}(\mathrm{i})}{\mathrm{d} \omega_{0}} S_{J m}\right|(+)_{J+m^{\prime}},(-)_{J-m^{\prime}}\right\rangle \otimes\right| n^{\prime}\right\rangle$,

Enfin, en constatant que l'action de l'opérateur symétrique

$$
\sum_{\mathrm{i}} U_{\mathrm{A}}^{+}(\mathrm{i}) \frac{\mathrm{d} U_{\mathrm{A}}(\mathrm{i})}{\mathrm{d} \omega_{0}}
$$

sur le vecteur symétrique $S_{J m}\left|(+)_{J+m^{\prime}},(-)_{J-m^{\prime}}>\otimes\right| n^{\prime}>$ est équivalent à $2 J$ fois celle de l'opérateur

on obtient :

$$
U_{\mathrm{A}}^{+}(1) \frac{\mathrm{d} U_{\mathrm{A}}(1)}{\mathrm{d} \omega_{0}},
$$

$\left\langle\overline{J m n} \mid \frac{\mathrm{d}}{\mathrm{d} \omega_{0}} \overline{J m^{\prime} n^{\prime}}\right\rangle=2 J \alpha_{J m} \alpha_{J m^{\prime}}\left\langle n\left|\otimes\left\langle(+)_{J+m},(-)_{J-m}\left|U_{\mathrm{A}}^{+}(1) \frac{\mathrm{d} U_{\mathrm{A}}(1)}{\mathrm{d} \omega_{0}} S_{J m}\right|(+)_{J+m^{\prime}},(-)_{J-m^{\prime}}\right\rangle \otimes\right| n^{\prime}\right\rangle$

L'opérateur $U_{\mathrm{A}}^{+}(1) \frac{\mathrm{d} U_{\mathrm{A}}(1)}{\mathrm{d} \omega_{0}}$ n'agit que sur le spin 1 et laisse tous les autres inchangés. Il en résulte que dans le développement du vecteur $S_{\mathrm{ym}} \mid(+)_{J+m^{\prime}},(-)_{J-m^{\prime}}>$, seuls les états pour lesquels les spins $2,3, \ldots 2 J$ sont rangés dans le même ordre que dans le vecteur $\mid(+)_{J+m},(-)_{J-m}>$ donnent une contribution non nulle à l'élément de matrice $\left\langle\overline{J m n} \mid \frac{\mathrm{d}}{\mathrm{d} \omega_{0}} \overline{J m^{\prime} n^{\prime}}\right\rangle$; il n'y a en effet que le premier spin qui peut se trouver dans deux états différents dans le bra et dans le ket car lui seul peut être affecté par l'opérateur

$$
U_{A}^{+}(1) \frac{d U_{A}(1)}{d \omega_{0}} .
$$

On en déduit que $\left\langle\overline{J m n} \mid \frac{\mathrm{d}}{\mathrm{d} \omega_{0}} \overline{J m^{\prime} n^{\prime}}\right\rangle$ ne peut être

différent de zéro que si $\left|m-m^{\prime}\right| \leqslant 1$.

Ce résultat important va nous permettre de déterminer l'ordre relatif des niveaux pour une valeur quelconque de $\Delta m=m-m^{\prime}$. Nous exclurons toutefois le cas $m-m^{\prime}=0$ (correspondant à deux niveaux parallèles du diagramme ne se croisant jamais) qui ne présente pas d'intérêt pour l'étude de l'ordre des croisements de niveaux.

Lorsque $m-m^{\prime}=+1$, on déduit immédiatement de (A.III. 10) l'équation

$$
\begin{aligned}
\left\langle\overline{J m n} \mid \frac{\mathrm{d}}{\mathrm{d} \omega_{0}} \overline{J, m-1, n^{\prime}}\right\rangle & =\frac{2 J}{2 J !} \alpha_{J m} \alpha_{J m^{\prime}}[J+m-1] ![J-m] !\left\langle+n\left|U_{\mathrm{A}}^{+} \frac{\mathrm{d} U_{\mathrm{A}}}{\mathrm{d} \omega_{0}}\right|-n^{\prime}\right\rangle \\
& =\frac{2 J[J+m-1] ![J-m] !}{\{[J+m] ![J-m] ![J+m-1] ![J-m+1] !\}^{1 / 2}}\left\langle\overline{+n} \mid \frac{\mathrm{d}}{\mathrm{d} \omega_{0}} \overline{-n^{\prime}}\right\rangle
\end{aligned}
$$

(le facteur $[J+m-1] ![J-m]$ ! correspond au nombre d'états qui dans la décomposition de

$$
S_{J m} \mid(+)_{J+m^{\prime}},(-)_{J-m^{\prime}}>
$$

ont leurs spins $2,3, \ldots 2 J$ occupant les mêmes états que dans $\mid(+)_{J+m},(-)_{J-m}>$ ). La relation (A.III.11), montre que dans le cas $\Delta m=1$, la quantité $\left\langle\overline{J m n} \mid \frac{\mathrm{d}}{\mathrm{d} \omega_{0}} \overline{J m^{\prime} n^{\prime}}\right\rangle$ est à un facteur numérique près, identique à celle que l'on calculerait pour un spin $\frac{1}{2}$ (une relation analogue peut être démontrée pour $\Delta m=m-m^{\prime}=-1$ ).

Envisageons à présent deux niveaux $\mid \overline{J m n}>$ et $\mid \overline{J m^{\prime} n^{\prime}}>$ de $\Delta m>1$. Pour déterminer leur ordre relatif, nous devons construire la chaîne

$$
\left\langle\overline{J m n} \mid \frac{\mathrm{d}}{\mathrm{d} \omega_{0}} \overline{J m_{1} n_{1}}\right\rangle\left\langle\overline{J m_{1} n_{1}} \mid \frac{\mathrm{d}}{\mathrm{d} \omega_{0}} \overline{J m_{2} n_{2}}\right\rangle \ldots\left\langle\overline{J m_{p} n_{p}} \mid \frac{\mathrm{d}}{\mathrm{d} \omega_{0}} \overline{J m^{\prime} n^{\prime}}\right\rangle
$$


d'ordre le plus bas permettant de les relier. Nous venons de montrer que chaque maillon de cette chaîne à un $\Delta m$ au plus égal à 1 . Il faut donc au moins $\left|m-m^{\prime}\right|-1$ états intermédiaires pour relier $\mid \overline{J m n}>$ à $\mid \overline{J m^{\prime} n^{\prime}}>$ : les deux niveaux sont d'ordre relatif au moins égal à $\left|m-m^{\prime}\right|-1$. En fait, les niveaux peuvent être d'un ordre relatif supérieur, par exemple infini, si les niveaux sont de symétries différentes.

Nous allons maintenant essayer de préciser cet ordre pour chaque polarisation de la radiofréquence.

a) CHAMP RF EN POLARisation $\pi$. - Les états propres $|\bar{J} m \bar{n}\rangle$ ne dépendent pas du champ $H_{0}$, tous les produits de la forme $\left\langle\overline{J m n} \mid \frac{\mathrm{d}}{\mathrm{d} \omega_{0}} \overline{J m^{\prime} n^{\prime}}\right\rangle$ sont nuls et l'ordre relatif d'un couple quelconque de niveaux est infini.

b) CHAMP RF EN POLARISATION $\sigma_{+} \cdot-$ Nous savons que si les deux niveaux $\mid \overline{J m n}>$ et $\mid \overline{J m^{\prime} n^{\prime}}>$ sont tels que leur $\Delta m+\Delta n$ est différent de zéro, ces niveaux sont de symétries différentes. Leur ordre relatif est alors infini. Par contre si $\Delta m+\Delta n=0$ les niveaux ont la même symétrie. On peut les relier par $\left|m-m^{\prime}\right|-1$ états intermédiaires, puisque chaque maillon (correspondant à un $\Delta m=-1$ et $\Delta n=+1$ )

$$
\left\langle\overline{J m n} \mid \frac{\mathrm{d}}{\mathrm{d} \omega_{0}} \overline{J m-1, n+1}\right\rangle
$$

est proportionnel à

$$
\left\langle\overline{+n} \mid \frac{\mathrm{d}}{\mathrm{d} \omega_{0}}-\overline{-}, n+1\right\rangle
$$

et se trouve alors effectivement différent de zéro (voir Appendice II relatif à l'ordre des niveaux $\mid \overline{+n}>$ et $1-, n+1>$ pour un $\left.\operatorname{spin} \frac{1}{2}\right)$.

L'ordre des niveaux est alors précisément égal à $\left|m-m^{\prime}\right|-1$.

c) CHAmp RF en polarisation $\sigma$. - Si les deux niveaux $|\overline{J m n}\rangle$ et $\left|\overline{J m^{\prime} n^{\prime}}\right\rangle$ ont un $\Delta m$ et un $\Delta n$ de parités opposées, ils sont de symétries différentes et leur ordre relatif est infini. Si $\Delta m$ et $\Delta n$ ont la même parité, les deux niveaux sont par contre de même symétrie. Il suffit que chaque maillon

$$
\left\langle\overline{J m n} \mid \frac{\mathrm{d}}{\mathrm{d} \omega_{0}} \overline{J, m-1, n^{\prime}}\right\rangle
$$

de la chaîne (A.III.12) change la parité de $n$ pour que ce maillon, proportionnel à $\left\langle\overline{+n} \mid \frac{\mathrm{d}}{\mathrm{d} \omega_{0}} \overline{-n^{\prime}}\right\rangle$ soit effectivement non nul (voir Appendice II relatif à l'ordre des niveaux $|+n\rangle$ et $\left|-n^{\prime}\right\rangle$ pour un spin $\left.\frac{1}{2}\right)$.

Il existe donc effectivement une chaîne à $\left|m-m^{\prime}\right|-1$ états intermédiaires, changeant un même nombre $\left(\left|m-m^{\prime}\right|\right)$ de fois la parité de $m$ et la parité de $n$ et permettant de relier les deux niveaux $|J m n\rangle$ et $\left|J m^{\prime} n^{\prime}\right\rangle$; ces derniers sont alors effectivement d'ordre relatif $\left|m-m^{\prime}\right|-1$. d) Champ RF en Polarisation COHÉRENTE QuelCONQUE. - Chaque maillon $\left\langle\overline{J m n} \mid \frac{\mathrm{d}}{\mathrm{d} \omega_{0}} \overline{J m-1, n^{\prime}}\right\rangle$ de la chaîne (A.III.12) proportionnel à

$$
\left\langle\overline{+n} \mid \frac{\mathrm{d}}{\mathrm{d} \omega_{0}} \overline{-n^{\prime}}\right\rangle
$$

est alors différent de zéro quel que soit $n^{\prime}$

$$
\left(\left\langle\overline{+n} \mid \frac{\mathrm{d}-n^{\prime}}{\mathrm{d} \omega_{0}}\right\rangle\right.
$$

est en effet non nul quels que soient $n$ et $n^{\prime}$ pour cette polarisation la plus générale possible de la RF). Il existe donc bien une chaîne à $\left|m-m^{\prime}\right|-1$ états intermédiaires reliant les niveaux $\mid \overline{J m n}>$ et $\mid \overline{J m^{\prime} n^{\prime}}>$ qui sont alors d'ordre $\left|m-m^{\prime}\right|-1$.

On peut résumer tous les résultats précédents de la façon suivante : deux niveaux quelconques $\mid \overline{J m n}>$ et $\mid \overline{J m^{\prime} n^{\prime}}>$ de l'atome habillé $\left(m \neq m^{\prime}\right)$ sont d'ordre relatif $\left|m-m^{\prime}\right|-1$, à moins qu'une raison de symétrie ne rende cet ordre infini.

Appendice IV : interprétation d'une résonance de croisement de niveaux de deuxième espèce en termes de processus d'absorption et d'émission de photons RF. - Pour simplifier au maximum la discussion, nous étudions le cas d'un niveau atomique excité de moment cinétique $J=1$, de largeur naturelle $\Gamma$, interagissant avec un champ RF de polarisation $\sigma$. L'atome est préparé dans le sous-niveau Zeeman $J=1, m=-1$ par excitation optique $\sigma^{-}$à partir du niveau fondamental $f$ de moment cinétique $J=0$; on mesure la probabilité de transfert $\mathfrak{T}_{-1,+1}$ vers le sous-niveau $J=1$, $m=+1$ en détectant la lumière $\sigma_{+}$réémise.

D'après les résultats du paragraphe 3 , les niveaux de l'atome habillé issus des états non perturbés $\mid-1, n>$ et $\mid+1, n>$ forment en $\omega_{0}=0$ un croisement de deuxième espèce d'ordre 1 . La résonance associée à ce croisement, que l'on observe en balayant $\omega_{0}$ autour de 0 , correspond à un transfert résonnant de population entre les états non perturbés $|-1, n\rangle$

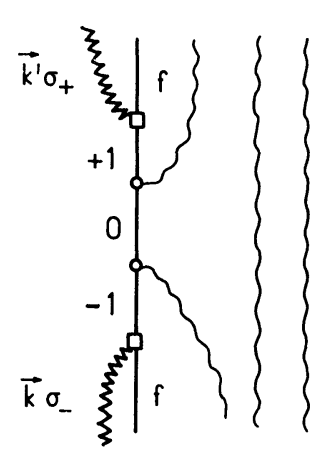

a)

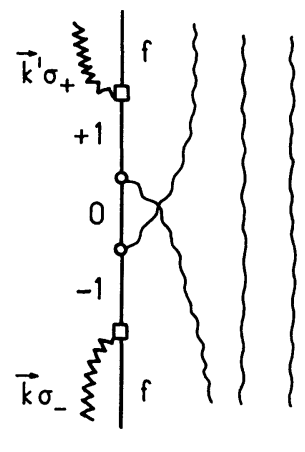

b)
FIG. 14. - Diagrammes schématisant à l'ordre le plus bas les amplitudes de diffusion d'un photon optique (ligne brisée) par un atome interagissant avec des photons RF (lignes ondulées). 
et $|+1, n\rangle$. Nous nous proposons d'interpréter cette résonance en termes de processus d'absorption et d'émission de photons RF par l'atome, en utilisant les notations et le formalisme développés dans la référence [3].

Le hamiltonien d'interaction couple à l'ordre le plus bas les états $|-1, n\rangle$ et $|+1, n\rangle$ aux états $|0, n+1\rangle$ et $|0, n-1\rangle$, les éléments de matrice étant tous égaux à une constante réelle $v$ (si l'on confond $\sqrt{n}$ et $\sqrt{n+1})\left({ }^{3}\right)$. Les deux diagrammes de Feynman qui, à l'ordre le plus bas, sont associés au processus de diffusion d'un photon optique $\left(\mathbf{k} \sigma_{-} \rightarrow \mathbf{k}^{\prime} \sigma_{+}\right)$par l'atome interagissant avec les photons RF sont représentés sur les figures $14 a$ et $14 b$ (comme dans la référence [3], les photons optiques sont représentés par des lignes brisées, les photons RF par des lignes ondulées). Vis-à-vis de la radiofréquence, les processus représentés en $14 a$ et $14 b$ sont des processus Raman qui ne diffèrent que par l'ordre dans lequel les photons sont absorbés et émis.

Les amplitudes de probabilité associées à $14 a$ et $14 b$ valent respectivement :

$$
\begin{aligned}
& B_{+}^{\prime} v^{2} B_{-} \frac{1}{E_{\mathrm{k}}-E_{0}-\omega_{0}+\frac{\mathrm{i} \Gamma}{2}} \frac{1}{E_{\mathrm{k}}-E_{0}+\omega+\frac{\mathrm{i} \Gamma}{2}} \frac{1}{E_{\mathrm{k}}-E_{0}+\omega_{0}+\frac{\mathrm{i} \Gamma}{2}} \\
& B_{+}^{\prime} v^{2} B_{-} \frac{1}{E_{\mathrm{k}}-E_{0}-\omega_{0}+\frac{\mathrm{i} \Gamma}{2}} \frac{1}{E_{\mathrm{k}}-E_{0}-\omega+\frac{\mathrm{i} \Gamma}{2}} \frac{1}{E_{\mathrm{k}}-E_{0}+\omega_{0}+\frac{\mathrm{i} \Gamma}{2}}
\end{aligned}
$$

$B_{-}$étant l'amplitude d'absorption d'un photon optique $\sigma_{-}$d'énergie $E_{\mathrm{k}}, B_{+}^{\prime}$ l'amplitude d'émission d'un photon $\sigma_{+}$. On voit que pour $E_{\mathrm{k}}$ voisin de $E_{0}$ et $\omega_{0}$ voisin de 0 , chacune des amplitudes (A.IV.1a) et (A.IV.1b) présente deux dénominateurs résonnants. Si l'on ne conservait qu'une seule de ces deux amplitudes, on obtiendrait en l'élevant au carré 4 dénominateurs résonnants et on se trouverait ainsi dans une situation identique à celle envisagée dans la référence [3] sous le nom de double résonance (conservation de l'énergie à l'issue de l'absorption du photon optique d'une part, à l'issue des transitions RF d'autre part).
En fait, pour $E_{\mathrm{k}}=E_{0}$ et $\omega_{0}=0$, les deux amplitudes (A.IV.1a) et (A.IV.1b) interfèrent quasi destructivement et le nombre de dénominateurs résonnants obtenus lorsqu'on élève au carré la somme de (A.IV .1a) et (A.IV.1b) passe de 4 à 2 .

Pour le voir de manière plus précise, développons

$$
\frac{1}{E_{\mathrm{k}}-E_{0} \pm \omega+\frac{\mathrm{i} \Gamma}{2}}
$$

au voisinage de $E_{\mathrm{k}}=E_{0}$. Il vient en ajoutant (A.IV.1a) et (A.IV.1b) :

$$
-\frac{2}{\omega^{2}} B_{+}^{\prime} v^{2} B_{-} \frac{E_{\mathrm{k}}-E_{0}+\frac{\mathrm{i} \Gamma}{2}}{\left(E_{\mathrm{k}}-E_{0}-\omega_{0}+\frac{\mathrm{i} \Gamma}{2}\right)\left(E_{\mathrm{k}}-E_{0}+\omega_{0}+\frac{\mathrm{i} \Gamma}{2}\right)} .
$$

On voit ainsi que pour $E_{\mathrm{k}} \sim E_{0}$ et $\omega_{0} \sim 0$, le numérateur de (A.IV.2) compense l'un des deux dénominateurs résonnants et qu'il ne reste plus alors dans l'amplitude globale qu'un seul dénominateur résonnant. L'interprétation de la résonance observée en terme de double résonance (optique puis Raman RF) n'est donc pas tout à fait valable.

On peut par contre réécrire (A.IV.2) pour $\omega_{0}<\Gamma$ et $E_{\mathbf{k}} \sim E_{0}$ sous la forme approchée :

$$
-\frac{B_{+}^{\prime} v^{2} B_{-}}{\omega^{2}} \frac{1}{E_{\mathrm{k}}-E_{0}-\omega_{0}+\mathrm{i} \frac{\Gamma}{2}}-\frac{B_{+}^{\prime} v^{2} B_{-}}{\omega^{2}} \frac{1}{E_{\mathrm{k}}-E_{0}+\omega_{0}+\frac{\mathrm{i} \Gamma}{2}}
$$

(3) Si l'on néglige tous les autres états du système global atome $+\mathrm{RF}$ et si l'on se limite aux 4 états $| \pm 1, n\rangle,|0, n \pm 1\rangle$, on se trouve dans une situation qui rappelle beaucoup celle du modèle simple présenté dans le paragraphe 2 de la référence [1], tant en ce qui concerne la variation avec $\omega_{0}$ des énergies non perturbées que la forme de la matrice représentant le couplage. C'est d'ailleurs l'étude de ce problème précis qui nous a suggéré le modèle simple de la référence [1]. ce qui permet de se représenter l'amplitude globale de diffusion comme la somme de deux termes associées aux diagrammes $15 a$ et $15 b$. Sur ces diagrammes, les lignes en traits renforcés représentent les états propres $|\overline{+1, n}\rangle$ et $|\overline{-1, n}\rangle$ de l'atome habillé calculés à l'ordre le plus bas en $v$ qui permettra l'absorption d'un photon $\sigma^{-}$et la réémission d'un photon $\sigma^{+}$.

Par exemple, dans $15 b$ l'état $\mid \overline{-1, n}>$ peut être 


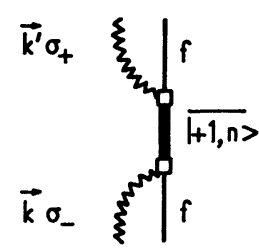

a)

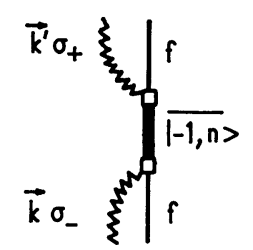

b)
Fig. 15. - Diagrammes schématisant les amplitudes de diffusion associées aux deux termes de A.IV.3. Les lignes en traits renforcés correspondent aux états $|\overline{+1, n}\rangle$ et $|\overline{-1, n}\rangle$ de l'atome habillé.

préparé à l'ordre 0 en $v$ par absorption d'un photon $\sigma_{-}$, mais il faut par contre considérer sa contamination en $-v^{2} / \omega^{2}$ par l'état $|+1, n\rangle$ (voir résultats $d u$ $\S 2$ de la référence [1] concernant les états propres perturbés relatifs au modèle simple) pour obtenir la réémission d'un photon $\sigma^{+}$; c'est ce qui explique le facteur $\left(B_{-}\right)\left(-\frac{v^{2}}{\omega^{2}} B_{+}^{\prime}\right)$ qui figure dans le deuxième terme de (A.IV.3). On peut donc considérer que la résonance observée sur $\mathfrak{T}_{-1,+1}$ provient d'une interférence entre deux amplitudes contenant chacune un dénominateur résonnant différent associé à un état propre différent de l'atome habillé.

REMARQUE. - Comme $\mathfrak{T}_{-1,+1}$ subit une variation résonnante, on pourrait croire que la différence de population entre les états -1 et +1 se comporte de la même façon et que le signal précédent est observable en orientation purre. En fait, on peut montrer qu'il existe également un transfert résonnant entre les niveaux $|-1, n\rangle$ et $|0, n \pm 1\rangle$ qui affecte également la population du niveau -1 . Il résulte du bilan global de ces processus que la différence de population entre les états -1 et +1 ne varie pas au voisinage du champ nul. Par conséquent, l'orientation atomique ne peut pas résonner. Dans l'expérience décrite ici (excitation en $\sigma_{-}$, détection en $\sigma_{+}$), on prépare et on détecte à la fois de l'orientation et de l'alignement atomique. Ce sont les variations de l'alignement qui sont responsables de la résonance observée sur $\mathcal{T}_{-1,+1}$. Nous décrivons dans la référence [15] une résonance en champ nul analogue, observable en alignement longitudinal.

\section{Bibliographie}

[1] Grynberg, G., Cohen-Tannoudj, C., DupontRoc, J. et Haroche, S., J. Physique 34 (1973) 523.

[2] Haroche, S., Ann. Phys. 6 (1971) 189 et 327.

[3] Cohen-TAnnoudi, et C. Haroche, S., J. mhysique 30 (1969) 125.

[4] Cohen-Tannoudi, C. et Haroche, S., J. Physique 30 (1969) 153.

[5] Winter, J. M., Thèse Paris (1958), Ann. Phys. 4 (1959) 745.

[6] Alexandrov, E. B., Constantinov, O. B., Pereli, B. I. et KHodovoy, B. A., J. Exp. Theoret. Phys. (USSR) 45 (1963) 503.

Favre, C. J. et Geneux, E., Phys. Lett. 8 (1964) 190.

Polonsky, N. et Cohen-Tannoudi, C., C. $R$. Hebd. Séan. Acad. Sci. 260 (1965) 5231 et C. R. Hebd. Séan. Acad. Sci. 261 (1965) 369.

[7] Cohen-Tannoudi, C. et Haroche, S., C. R. Hebd. Séan. Acad. Sci. 261 (1965) 5400.

[8] Le Dourneuf, M., Thèse de $3^{\mathrm{e}}$ cycle Paris (1971).
[9] Dodd, J. N. et Series, G. W., Proc. Roy. Soc. A 263 (1961) 353.

Dodd, J. N., Series, G. W. et Taylor, M. J., Proc. Roy. Soc., A 273 (1963) 41.

[10] Cohen-Tannoudji, C. et Haroche, S., C. R. Hebd. Séan. Acad. Sci. 264 (1967) 626.

[11] Le Dourneuf, M., Cohen-Tannoudi, C., DupontRoc, J. et Haroche, S., C. R. Hebd. Séan. Acad. Sci. 272 (1971) 985.

[12] Majorana, E., Nuevo Cimento 9 (1932) 43.

[13] Cagnac, B., Thèse Paris (1961), Ann. Phys. 6 (1961) 467.

[14] Grynberg, G., Thèse de $3^{\mathrm{e}}$ cycle Paris (1972).

[15] Grynberg, G., Cohen-Tannoudj, C., DupontRoc, J. et Haroche, S., Opt. Commun. 6 (1972) 146.

[16] Messiah, A., Mécanique quantique Tome II (Dunod, Paris) 1964. 